\title{
A COHOMOLOGICAL APPROACH TO THE BRAUER-LONG GROUP AND THE GROUPS OF GALOIS EXTENSIONS AND STRONGLY GRADED RINGS
}

\author{
S. CAENEPEEL AND M. BEATTIE
}

\begin{abstract}
Let $G$ be a finite abelian group, and $R$ a commutative ring. The Brauer-Long group $\mathrm{BD}(R, G)$ is described by an exact sequence

$$
1 \rightarrow \mathrm{BD}^{s}(R, G) \rightarrow \mathrm{BD}(R, G) \stackrel{\beta}{\rightarrow} \operatorname{Aut}\left(G \times G^{*}\right)(R)
$$

where $\operatorname{BD}^{s}(R, G)$ is a product of étale cohomology groups, and $\operatorname{Im} \beta$ is a kind of orthogonal subgroup of $\operatorname{Aut}\left(G \times G^{*}\right)(R)$. This sequence generalizes some other well-known exact sequences, and restricts to two split exact sequences describing Galois extensions and strongly graded rings.
\end{abstract}

\section{INTRODUCTION}

In 1964, Wall introduced a Brauer group of classes of Z/2Z-graded algebras over a field with multiplication induced by a twisted tensor product, to study the Witt ring of quadratic forms (cf. [37]). This group was named the BrauerWall group, and, since then, some various generalizations to commutative rings and to finite abelian groups have been introduced by Bass and Small [2, 31], Knus [20], and Childs, Garfinkel and Orzech [13]. In [23], Long introduced a Brauer group consisting of equivalence classes of $G$-dimodule algebras over a commutative ring $R$, and for an arbitrary abelian group $G$. This new invariant of $R$ and $G$ contained all the previous generalizations of the Brauer-Wall group as subgroups, and, moreover, it contains other invariants of $R$, namely the Brauer group of $R$, the Galois extensions of $R$ with group $G$, the strongly $G$-graded rings having $R$ as part of degree zero, and the automorphism group of $G$. Afterwards, this new Brauer group was called the Brauer-Long group, denoted by $\operatorname{BD}(R, G)$. In the literature, many calculations have been done in some special cases; some authors restricted attention to some subgroups of the Brauer-Long group $[3,4,6,14,28]$, while others considered only the case where $G$ is cyclic $[7,16]$ or a product of cyclic groups of the same order [12]. In most

Received by the editors March 29, 1989.

1980 Mathematics Subject Classification (1985 Revision). Primary 13A20, 16A16, 13 B05.

Key words and phrases. Brauer group, Azumaya algebra, Galois extension, étale cohomology, strongly graded ring, dimodule algebra.

The research of the first author was supported by the Belgian National Science Foundation NFWO and that of the second author by the Natural Sciences and Engineering Research Council of Canada. 
cases some more restrictions were put on the ground ring $R$ : the Picard group of $R$ was supposed to be trivial, $R$ was supposed to contain "enough" roots of unity, and $|G|$ was supposed to be invertible in $R$.

So far, no general approach has been proposed. Also, most authors have studied the Brauer-Long group from a ring theoretic point of view, not examining the question whether there exists a dimodule analog to Gabber's theorem giving the relation between the Brauer group and the second étale cohomology group of the sheaf of units (cf. [18]). In this paper, we present such a cohomological interpretation of the Brauer-Long group. Our approach is merely based on descent theory, as presented by Knus and Ojanguren in [22], and is also inspired by the Z-graded cohomology developed by the first author and F. Van Oystaeyen in [9].

To overcome difficulties concerning the nontriviality of the Picard group, we introduce a dimodule version of the Picard group, $\operatorname{PD}(R, G)$ in $\S 1$. It is not very difficult to see that the sheaf associated to $\operatorname{PD}(\cdot, G)$ on the étale site is not trivial, actually, it is given by the sheaf $G \times G^{*}(\cdot)$. Also, the sheaf associated to $\operatorname{BD}(\cdot, G)$ is not trivial, since the Brauer-Long group of a strictly Henselian ring is not trivial. As a consequence, there is no splitting theorem for the BrauerLong group, and this has led us to first consider $\operatorname{BD}^{s}(R, G)$, the subgroup of classes split by some faithfully flat extension $S$ of $R$. In $\S 2$, it is shown that $\mathrm{BD}^{S}(R, G)$ is isomorphic to a semidirect product of $H^{2}\left(R_{\text {et }}, \mathbf{G}_{\mathrm{m}}\right)_{\text {tors }}$ and $H^{1}\left(R_{\text {ét }}, G \times G^{*}\right)$, where the multiplication rules are defined by a kind of cup product map. The $H^{1}$ factor is caused by the nontriviality of the sheaf associated to $\operatorname{PD}(\cdot, G)$.

To describe the complete Brauer-Long group, we define a map $\beta: \mathrm{BD}(R, G)$ $\rightarrow \operatorname{Aut}\left(G \times G^{*}\right)(R)$, such that $\operatorname{Ker} \beta=\operatorname{BD}^{S}(R, G)$. To define $\beta$, we use an appropriate version of an exact sequence due to Rosenberg and Zelinsky which generalizes the Skolem-Noether Theorem. This generalizes maps considered by the second author, Childs and Orzech $[4,12,28]$. The image of $\beta$ is shown to be a kind of orthogonal subgroup of $\operatorname{Aut}\left(G \times G^{*}\right)(R)$. This describes the BrauerLong group, up to the exact multiplication rules in the product $\operatorname{BD}^{s}(R, G) \times$ $\operatorname{Im} \beta$. These rules are known in the special case where $G$ is cyclic (cf. $[7,16]$ ).

In previous papers, the second author tried to describe the Brauer-Long group using Galois theory. In $\S 4$, we do the converse: the exact sequence obtained in $\S 3$ restricts to split exact sequences describing the group of Galois extensions, and the group of $G$-strongly graded rings having $R$ as their part of degree zero. This generalizes an exact sequence of DeMeyer. We also provide a cohomological description of the groups of commutative Galois extensions and commutative strongly graded rings, and present a generalization of the Kümmer exact sequence to finite abelian groups. In the last section of this paper, we apply our results to describe the graded Brauer group of Childs, Garfinkel and Orzech. This will generalize the results of [12].

Throughout this paper, we impose no restrictions on $R$ and $G$, concerning the connectedness of $R$, the Picard group of $R$, cyclicness of $G$ and existence 
of roots of unity. However, it will turn out that the structure of $\operatorname{BD}(R, G)$ is the richest under the conditions that we have enough roots of unity and that $|G|$ is invertible, and therefore we impose these conditions in some of our propositions. We expect that the methods developed in this paper can also be used to describe the Brauer-Long group with respect to a Hopf algebra, as introduced in [24]. This will be discussed in a forthcoming paper.

\section{NOTATION AND PRELIMINARY RESULTS}

1.1. Dimodules and dimodule algebras. Let $R$ be a commutative ring, and $G$ an abelian group (in most of our applications, $G$ will be finite), denoted additively (in this context, it is common to denote $G$ multiplicatively; it will turn out however that, for our results, the formalism becomes clearer if we denote $G$ additively). A $G$-module $M$ is an $R$-module $M$ with an action of $G$ on $M$ satisfying $(\sigma+\tau) m=\sigma(\tau m)$ and $(o) m=m$, for all $m \in M$, $\sigma, \tau \in G$. A $G$-dimodule $M$ is a $G$-graded module which is also a $G$-module such that the action and grading are compatible, that is $\sigma\left(M_{\tau}\right)$ is contained in $M_{\tau}$, for all $\sigma, \tau \in G$. A $G$-module algebra $A$ is an $R$-algebra which is a $G$ module such that $\sigma(a b)=\sigma(a) \sigma(b)$, for all $\sigma \in G, a, b \in A$. A $G$-dimodule algebra is a $G$-graded algebra which is a $G$-dimodule and a $G$-module algebra.

Given two $G$-(di)modules $M, N, \operatorname{Hom}_{R}(M, N)$ can be given a $G$-module structure by defining $(\sigma f)(m)=\sigma(f((-\sigma) m))$, for all $m \in M, \sigma \in G, f \in$ $\operatorname{Hom}_{R}(M, N)$. Also $\operatorname{Hom}_{R}(M, N)$ can be given a grading in the natural way (cf. [27] for further details about graded rings), and this gives $\operatorname{Hom}_{R}(M, N)$ a $G$-dimodule structure. Unless it is specified otherwise, we will assume that $\operatorname{Hom}_{R}(M, N)$ is furnished with this natural $G$-dimodule structure. The tensor product $M \otimes_{R} N$ of two $G$-dimodules has a natural dimodule structure in the following way: $\sigma(m \otimes n)=\sigma(m) \otimes \sigma(n)$ for the module structure, and $\left(M \otimes_{R} N\right)_{\sigma}=\bigoplus_{\tau \in G} M_{\sigma-\tau} \otimes M_{\tau}$ for the grading. The tensor product of two $G$-dimodule algebras $A, B$ is again a $G$-dimodule algebra. We define the smash product $A \#_{R} B$ of $A$ and $B$ to be $A \otimes_{R} B$ as a $G$-dimodule, but with multiplication defined by

$$
\left(a_{1} \# b_{1}\right)\left(a_{2} \# b_{2}\right)=a_{1} \beta_{1}\left(a_{2}\right) \# b_{1} b_{2}
$$

where $a_{i} \in A, b_{i} \in B$ and $b_{1}$ is homogeneous of grade $\beta_{1} . a \otimes b$ is denoted $a \# b$ when viewed as an element of $A \# B$. Given two homogeneous morphisms $f_{i}: N_{i} \rightarrow P_{i}(i=1,2)$, we define the smash product $f_{1} \# f_{2}: N_{1} \otimes N_{2} \rightarrow P_{1} \otimes P_{2}$ by

$$
\left(f_{1} \# f_{2}\right)\left(n_{1} \otimes n_{2}\right)=f_{1}\left(\varphi_{2}\left(n_{1}\right)\right) \otimes f_{2}\left(n_{2}\right)
$$

where $\operatorname{deg} f_{2}=\varphi_{2}$. This rule is extended to nonhomogeneous morphisms by linearity. It is an easy exercise to prove that (1.1.1) also holds for the composition of homogeneous morphisms, that is, if $g_{i}: M_{i} \rightarrow N_{i}$, with $\operatorname{deg} g_{i}=\gamma_{i}$, 
then

$$
\left(g_{1} \# g_{2}\right) \circ\left(f_{1} \# f_{2}\right)=g_{1} \circ \gamma_{2}\left(f_{1}\right) \# g_{2} \circ f_{2} .
$$

Also, if $a$ and $b$ are invertible homogeneous elements or morphisms of degree $\alpha$ and $\beta$, then

$$
(a \# b)^{-1}=(-\beta)\left(a^{-1}\right) \# b^{-1} .
$$

A homomorphism between $G$-dimodules that preserves the action and the grading is called a $G$-dimodule homomorphism. The category of $R-G$-dimodules and $R$-G-dimodule homomorphisms is denoted by $(R, G)$-dimod. The fact that two $R$-G-dimodules are isomorphic is denoted by $M \cong{ }_{d} N$.

1.2. Consider $R_{\mathrm{Zar}}, R_{\text {ét }}$ or $R_{\mathrm{fl}}$ (cf. [26] for all details on sites), and let $G$ be the constant presheaf on any of these sites. Let $G(\cdot)$ be the sheaf associated to $G$ : for $R \rightarrow S$ in $R_{\mathrm{Zar}}, R_{\text {ét }}$ or $R_{\mathrm{fl}}, G(S)=\{\sigma: \operatorname{Spec}(S) \rightarrow G \mid \sigma$ is continuous $\}=$ $\{\sigma: \operatorname{Spec}(S) \rightarrow G \mid \sigma$ is locally constant $\}$. Now let $M$ be a $G$-dimodule. Then $M$ has a $G(R)$-dimodule structure defined as follows: $\operatorname{deg}_{G(R)}(m)=$ $\sigma$ if for all $p \in \operatorname{Spec}(R): \operatorname{deg}_{G}\left(m_{p}\right)=\sigma(p)$, where $m_{p}$ is the image of $m$ in $M_{p}$; to define an action, consider a direct sum decomposition $R=R_{1} \oplus$ $\cdots \oplus R_{n}$ such that $\sigma \in G(R)$ takes the constant value $\sigma_{i}$ on $\operatorname{Spec}\left(R_{i}\right)$. For $m \in R_{i} M$, we define $\sigma m=\sigma_{i} m$. The dual sheaf of $G(\cdot)$ is denoted $G^{*}(\cdot)$, that is $G^{*}(S)=\operatorname{Hom}\left(G(S), \mathbf{G}_{\mathbf{m}}(S)\right)$, where $\mathbf{G}_{\mathbf{m}}$ is the multiplicative sheaf of units. Take $\left(\sigma, \sigma^{*}\right) \in\left(G \times G^{*}\right)(R)$; we define a $G$-dimodule $R\left(\sigma, \sigma^{*}\right)$ as follows: $R\left(\sigma, \sigma^{*}\right)$ is $R$ as an $R$-module; take a direct summand $T$ of $R$ on which $\left(\sigma, \sigma^{*}\right)$ takes constant values $\left(\tau, \tau^{*}\right)$. The grading on $T$ is defined by giving every element of $T$ degree $\tau$, and the action by $\rho(t)=\tau^{*}(\rho) t$ for every $\rho \in G, t \in T$. Note that the operation on $G^{*}$ is denoted additively, i.e. for $\sigma^{*}, \tau^{*} \in G^{*}, \rho \in G$, we have $\left(\sigma^{*}+\tau^{*}\right)(\rho)=\sigma^{*}(\rho) \tau^{*}(\rho)$.

The identity map $i: R \rightarrow R\left(\sigma, \sigma^{*}\right)$ is not a dimodule isomorphism, but is homogeneous of degree $\sigma$. The action of $G$ on $i$ and $i^{-1}$ is given by $\rho i=\sigma^{*}(\rho) i$ and $\rho\left(i^{-1}\right)=\sigma^{*}(-\rho) i^{-1}$. Let $(R, G)$-gr be the category of $G$ graded $R$-modules and graded homomorphisms (cf. [27]); we define a functor $\left(R, G \times G^{*}\right)$-gr $\rightarrow(R, G)$-dimod as follows: given a $G \times G^{*}$-graded $R$-module $M$, we define a $G$-dimodule structure on $M$ as follows: take $m \in M$ homogeneous of degree $\left(\sigma, \sigma^{*}\right)$; then $\operatorname{deg}_{G}(m)=\sigma$, and $\tau(m)=\sigma^{*}(\tau) m$, for all $\tau \in G$.

1.3. Proposition. If the canonical map $G(R) \rightarrow G^{* *}(R)$ is an isomorphism, and if $|G|$ is invertible in $R$, then the categories $\left(R, G \times G^{*}\right)$-gr and $(R, G)$-dimod are equivalent.

Note. From the fact that $|G|^{-1} \in R$, it follows that $G(R) \rightarrow G^{* *}(R)$ is injective; then the fact that $G(R) \rightarrow G^{* *} R$ is surjective means that every component of $R$ contains a primitive $\exp (G)$ th root of unity.

Proof. We refer to $[12, \S 5]$. 
We denote by $\operatorname{FPD}(R, G)$ the category of faithfully projective $R$ - $G$-dimodules and $R$ - $G$-dimodule homomorphisms. $\operatorname{PD}(R, G)$ will be the category of invertible $R$ - $G$-dimodules and $R$-G-dimodule homomorphisms. $\operatorname{PD}(R, G)=$ $K_{0} \mathbf{P D}(R, G)$ is the group of isomorphism classes of invertible $G$-dimodules, and we call this the dimodule Picard group of $R$. The tensor product is a product on these categories. We use similar notations for the subcategories $\operatorname{FPC}(R, G), \operatorname{PC}(R, G), \operatorname{FPM}(R, G), \operatorname{PM}(R, G)$ consisting of respectively $G$-graded modules and $G$-modules.

1.4. Lemma. Let $R$ be a commutative ring, and let $G$ be a finite abelian group. (1.4.1) $\mathrm{PD}(R, G) \cong \operatorname{Pic}(R) \times G(R) \times G^{*}(R)$;

(1.4.2) $\mathrm{PC}(R, G) \cong \operatorname{Pic}(R) \times G(R)$;

(1.4.3) $\mathrm{PM}(R, G) \cong \operatorname{Pic}(R) \times G^{*}(R)$.

Proof. Using the notation introduced in 1.2, we obtain a monomorphism $i$ : $G(R) \times G^{*}(R) \rightarrow \operatorname{PD}(R, G)$, defined by $i\left(\sigma, \sigma^{*}\right)=R\left(\sigma, \sigma^{*}\right)$. Consider an invertible $R$-dimodule $I$, and $p \in \operatorname{Spec}(R)$. As an $R_{p}$-module, $R_{p} \cong I_{p}$, and therefore $I_{p}$ is just $R_{p}$ as an $R_{p}$-module, but with grading given by $\operatorname{deg}(x)=\sigma_{p}$ for some $\sigma_{p} \in G$, and for all $x \in I_{p}$. The map $\operatorname{Spec}(R) \rightarrow G: p \rightarrow \sigma_{p}$ is clearly locally constant, and determines an element $\sigma$ of $G(R)$. We define $\sigma^{*} \in G^{*}(R)$ as follows: for $p \in \operatorname{Spec}(R)$, we let $1_{p}$ be the image of 1 under the isomorphism $R_{p} \cong I_{p}$. For $\tau \in G(R)$, we define $\sigma^{*}(\tau)$ by $\sigma^{*}(\tau)_{p}=\tau(p)\left(1_{p}\right)$. Because $\tau$ is locally constant, it is easily seen that $\sigma^{*}(\tau)_{p}$ globalizes to $\sigma^{*}(\tau) \in R$. The map $\left(e, e^{*}\right): \operatorname{PD}(R, G) \rightarrow G(R) \times G^{*}(R)$, defined by $I \rightarrow\left(\sigma, \sigma^{*}\right)$ is an epimorphism split by $i$, and $\operatorname{Ker}\left(e, e^{*}\right)=\operatorname{Pic}(R)$. This proves (1.4.1), and the two other statements follow immediately.

1.5. Notation. In the sequel, $S$ will always be a commutative $R$-algebra. Unless otherwise indicated, the grading and action of $G$ on $R$ and $S$ will be trivial, and all tensor and smash products will be over $R . S^{(n)}$ will be an abbrevation for the tensor product of $n$ copies of $S$ over $R$, and $\otimes_{n}$ and $\#_{n}$ will denote the tensor and smash product over $S^{(n)}$. If $M$ is an $R$-module, then we denote $M_{1}=S \otimes M, M_{2}=M \otimes S, M_{11}=S \otimes S \otimes M$, etc. For a homomorphism $f: M_{1} \otimes \cdots \otimes M_{n} \rightarrow M_{1}^{\prime} \otimes \cdots \otimes M_{n}^{\prime}, f_{i}$ will be the homomorphism $f_{i}: M_{1} \otimes \cdots \otimes S \otimes \cdots \otimes M_{n} \rightarrow M_{1}^{\prime} \otimes \cdots \otimes S \otimes \cdots \otimes M_{n}^{\prime}$, obtained by tensoring up in the $i$ th position. With this notation, we have

1.6. Lemma (faithfully flat descent for dimodules). Let $S$ be a faithfully flat commutative $R$-algebra, and $M$ an $S$-G-dimodule. Suppose that $u: M_{1} \rightarrow M_{2}$ is an $S^{(2)}$-G-dimodule isomorphism, and a descent datum, i.e. $u_{2}=u_{3} u_{1}$. Then there exists an $R$-G-dimodule $N$, and a dimodule isomorphism $\eta: N \otimes S \rightarrow$ $M$ such that the following diagram of $S^{(2)}$-dimodule isomorphisms commutes 
( $\tau$ denotes the switch map):

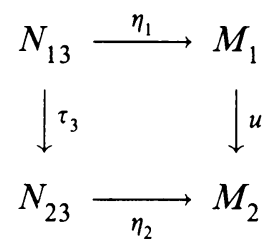

The pair $(N, \eta)$ is unique up to dimodule isomorphism. If $M$ is a dimodule algebra, then $N$ has a unique $R$-dimodule algebra structure such that $\eta$ is an $S$-dimodule algebra isomorphism. Similar statements hold for G-modules and G-graded modules.

Proof. Observe that the descended module (algebra) defined in [22, 3.2-3.4] has a natural structure of $G$-dimodule ( $G$-dimodule algebra).

1.7. Lemma. Let $P, Q \in \mathbf{F P D}(R, G)$ and $\alpha: \operatorname{End}_{R}(P) \rightarrow \operatorname{End}_{R}(Q)$ an $R$-Gdimodule algebra isomorphism. Then there exists $I \in \mathbf{P D}(R, G)$ and a dimodule isomorphism $f: P \otimes I \rightarrow Q$ such that $f$ induces $\alpha$, that is $\alpha(h)=f(h \otimes 1) f^{-1}$. $f$ and $I$ are unique up to dimodule isomorphism. Similar statements hold for $G$-module and G-graded module isomorphisms.

Proof. The Morita equivalences [17, I.3.3] are directly generalizable to the corresponding categories of dimodules. Then the result follows immediately by copying the proof of $[22$, IV.1.3].

1.8. G-Azumaya algebras and the Brauer-Long group. Let $A$ be an $R$ - $G$-dimodule algebra. The $G$-opposite algebra $\bar{A}$ of $A$ is defined to be $A$ as a $G$-dimodule, but with multiplication given by $a \cdot b=(\operatorname{deg} a)(b) a$. An $R-G$ dimodule algebra is called an $R$-G-Azumaya algebra if the following properties hold:

(1.8.1) $A$ is faithfully projective as an $R$-module;

(1.8.2) The maps $F: A \# \bar{A} \rightarrow \operatorname{End}_{R}(A)$ and $G: \bar{A} \# A \rightarrow\left(\operatorname{End}_{R}(A)\right)^{\text {opp }}$, given by $F(a \# b)(c)(a \# b)(c)=a \beta(c) b$ and $G(a \# b)(c)=\gamma(a) c b$ (where $\operatorname{deg} b=\beta$, $\operatorname{deg} c=\gamma)$ are isomorphisms of dimodule algebras.

It may be shown (cf. $[23,24]$ ) that the smash product of two $G$-Azumaya algebras is again $G$-Azumaya, and that the endomorphism ring of a faithfully projective $G$-dimodule is a $G$-Azumaya algebra. Also the $G$-opposite of a $G$ Azumaya algebra is $G$-Azumaya. Two $G$-Azumaya algebras $A, B$ are called Brauer equivalent if there exist faithfully projective $G$-dimodules $M, N$ such that $A \# \operatorname{End}_{R}(M) \cong_{d} B \# \operatorname{End}_{R}(N)$. The set of equivalence classes of this relation forms a group under the operation induced by the smash product. The inverse class of a class $[A]$ is represented by the $G$-opposite algebra of $A$. For details concerning this definition, we refer to [23, 24]. Following [16], we call this group the Brauer-Long group of $R$ with respect to $G$, and we denote it by $\mathrm{BD}(R, G)$.

1.9. Proposition. Let $A$ be an $R$-G-dimodule algebra. Then $A$ is a $G$-Azumaya algebra if and only if $A$ is a separable left and right $G$-central algebra, that is 
$\{a \in h(A): a b=\alpha(b) a$ for all $b \in B\}=R=\{a \in A: b a=\beta(a) b$ for all $b \in h(B)\}$ (we denoted $\operatorname{deg} a=\alpha, \operatorname{deg} b=\beta$, and $h(A)$ for the set of homogeneous elements of $A$ ).

Proof. If $G(R) \cong G^{* *}(R)$ and if $|G|$ is invertible in $R$, then the proposition follows from [13, 2.8] and [12, §5]. In general, one has to adapt the proof of [22, III.5.1], as was pointed out to us by F. Tilborghs [34].

If $R$ is not noetherian, then $G(R)$ can be an infinite group, and this may cause technical problems. The following lemma illustrates that it is possible to descend to noetherian subrings:

1.10. Lemma. Suppose that $R$ is a nonnoetherian commutative ring, and that $G$ is a finite abelian group such that $|G|$ is invertible in $R$ and $G(R) \cong G^{* *}(R)$. Then for any $R$-G-Azumaya algebra $A$, there exists a noetherian subring $R^{\prime}$ of $R$ and a $R^{\prime}$-G-Azumaya algebra $A^{\prime}$ such that $A \cong{ }_{d} A^{\prime} \otimes R$.

Proof. First, let $P$ be a faithfully projective $G$ - $R$-dimodule. Then $P$ is $\left(G \times G^{*}\right)(R)$-graded, so $P$ is a graded direct summand of a $\left(G \times G^{*}\right)(R)$-graded free $R$-module (cf. [27]). Thus $P$ is a direct summand of a module of the form

$$
\prod_{i=1}^{n} R\left(\sigma_{i}, \sigma_{i}^{*}\right) \text {. }
$$

Now, [22, I.2.8-I.2.9] may be generalized to $G$-dimodule homomorphisms: $G$ dimodule homomorphisms between faithfully projective dimodules may be descended to $G$-dimodule homomorphisms over a noetherian subring. Then [22, III.5.7] can be generalized directly.

1.11. Étale cohomology. Let $F$ be a sheaf on $R_{\mathrm{ff}}$ or $R_{\mathrm{et}}$. The sheaf cohomology groups on these sites will be denoted by $H^{n}(R, F)$. We refer to [26] for their definition. Artin ([1]) proved that the étale cohomology groups may be described by Amitsur cohomology:

$$
H^{n}\left(R_{\text {ét }}, F\right) \cong \lim _{\longrightarrow} H^{n}(S / R, F)
$$

where the inductive limit runs over all etale coverings $S$ of $R$. Concerning Amitsur cohomology, we will use the notations introduced in [22]. In particular, for $x \in F\left(S^{(n)}\right), x_{i}$ will denote $F\left(\varepsilon_{i}\right)(x)$, where $\varepsilon_{i}: S^{(n)} \rightarrow S^{(n+1)}: s_{1} \otimes \cdots \otimes$ $s_{n} \rightarrow s_{1} \otimes \cdots \otimes 1 \otimes \cdots \otimes s_{n}$, as usual.

1.12. Galois objects. Recall (cf. [3]) that for $H$ a finitely generated projective commutative and cocommutative Hopf algebra, $\operatorname{Gal}(R, H)$ is defined to be the abelian group of (not necessarily commutative) Galois $H$-objects in the sense of Chase and Sweedler [11, p. 56]. If $H=R G$, then $\operatorname{Gal}(R, R G)$ is the set of isomorphism classes of strongly $G$-graded $R$-algebras $T$ with $T_{0}=R$. Multiplication in $\operatorname{Gal}(R, R G)$ is defined by $S T=\bigoplus_{\sigma \in G} S_{\sigma} \otimes T_{\sigma}$. If $H=G R$, the dual of the group ring, then $\operatorname{Gal}(R, G R)$ is the set of Galois extensions of $R$ with group $G$ in the sense of [15]. Multiplication on $\operatorname{Gal}(R, G R)$ is defined by $S T=\{x \in S \otimes T:(\sigma \otimes 0) x=(0 \otimes \sigma) x$ for all $\sigma \in G\}$. 
Let $\mathrm{Gal}^{s}(R, G R)$ be the subgroup of $\operatorname{Gal}(R, G R)$ consisting of commutative objects. From Chase and Sweedler's definition of commutative Galois objects, or from [17, III.1.2], it then follows that

$$
\begin{aligned}
& \operatorname{Gal}^{S}(R, G R)=\{S \in \operatorname{Gal}(R, G R): S \otimes \underline{S} \cong G \underline{S}\} \\
& \quad=\{S \in \operatorname{Gal}(R, G R): S \otimes T \cong G T \text { for some étale covering } T \text { of } R\} .
\end{aligned}
$$

Here the isomorphisms are $G$-module algebra isomorphisms, and $\underline{S}$ is $S$ with trivial $G$-action. Remark that $\mathrm{Gal}^{s}(R, G R)$ is the familiar group $T(R, G)$ of Harrison [19]. Similarly, if we let $\operatorname{Gal}^{S}(R, R G)$ be the subgroup of $\operatorname{Gal}(R, R G)$ consisting of commutative strongly graded rings, then

$$
\begin{aligned}
\mathrm{Gal}^{S}(R, R G)=\{S \in \mathrm{Gal}(R, R G): S \otimes \underline{S} \cong \underline{S} G\} \\
\quad=\{S \in \mathrm{Gal}(R, R G): S \otimes T \cong T G \text { for some faithfully flat } R \text {-algebra } T\} .
\end{aligned}
$$

Now the isomorphisms are $G$-graded algebra isomorphisms, and $\underline{S}$ is $S$, but with trivial grading.

\subsection{Proposition. We have the following isomorphisms of groups:}

$\mathrm{Gal}^{s}(R, G R) \cong H^{1}\left(R_{\mathrm{fl}}, G\right) \cong H^{1}\left(R_{\text {ét }}, G\right)$ and $\operatorname{Gal}^{s}(R, R G) \cong H^{1}\left(R_{\mathrm{fl}}, G^{*}\right)$. Proof. This is an immediate consequence of [22, Proposition 2.8.1]. Let $S$ be a faithfully flat extension of $R$. Then the torsors (formes tordues) of $G R$ for the extension $S$ are classified by $H^{1}(S / R, A)$, where $A(T)$ is the group of $G$-module algebra automorphisms of $G T$. It is easy to show that $A(T) \cong G(T)$. Similarly, the torsors of $R G$ under the extension $S$ are classified by $H^{1}(S / R, A)$, where now $A(T)$, the group of $G$-graded algebra automorphisms of $T G$, is equal to $G^{*}(T)$. Taking inductive limits over all étale or flat coverings, the result follows. The fact that the connecting maps are homomorphisms can be proved in a straightforward way; for full detail, we refer to [9, VI.1.9-VI.1.10] or to [26, III.4].

1.14. Corollary. Every element in $H^{1}\left(R_{\mathrm{et}}, G\right)\left(H^{1}\left(R_{\mathrm{fl}}, G^{*}\right)\right)$ can be represented by a cocycle in $Z^{1}(S / R, G)\left(Z^{1}\left(S / R, G^{*}\right)\right)$ for some faithfully projective extension $S$ of $R$.

Proof. $S \in \mathrm{Gal}^{S}(R, G R)\left(\mathrm{Gal}^{S}(R, R G)\right)$ is split by $\underline{S}$, and $\underline{S}$ is clearly faithfully projective.

\section{The SPLit PART OF THE BRAUER-LONG GROUP}

One of the difficulties that we encounter studying the Brauer-Long group is the fact that the Brauer-Long group of a strictly Henselian ring is not trivial. Therefore we first restrict to the study of the subgroup $\operatorname{BD}^{S}(R, G)$ of elements represented by algebras which can be split by a faithfully flat extension of the ground ring:

$$
\mathrm{BD}^{S}(R, G)=\bigcup\{\mathrm{BD}(S / R, G): S / R \text { is faithfully flat }\}
$$


where

$$
\mathrm{BD}(S / R, G)=\operatorname{Ker}(\mathrm{BD}(R, G) \rightarrow \mathrm{BD}(S, G)) .
$$

In this section, we give a cohomological description of $\operatorname{BD}^{s}(R, G)$. First, we define a map which is, in a sense, a generalization of the cup product map (cf. $[16,26])$. Let $S$ be a faithfully flat $R$-algebra and consider the bilinear map

$$
\varphi: G^{*}\left(S^{(2)}\right) \times G\left(S^{(2)}\right) \rightarrow \mathbf{G}_{\mathbf{m}}\left(S^{(3)}\right)
$$

defined by

$$
\varphi\left(\sigma^{*}, \sigma\right)=\sigma_{1}^{*}\left(\sigma_{3}\right) .
$$

2.1. Lemma. The map $\varphi$ defined above induces well-defined maps (still denoted by $\varphi)$ :

$$
\varphi: H^{1}\left(S / R, G^{*}\right) \times H^{1}(S / R, G) \rightarrow H^{2}\left(S / R, \mathbf{G}_{\mathbf{m}}\right),
$$

and

$$
\varphi: H^{1}\left(R, G^{*}\right) \times H^{1}(R, G) \rightarrow H^{2}\left(R, \mathbf{G}_{\mathbf{m}}\right),
$$

where the cohomology may be taken on the flat or étale site.

Proof. First, we show that, if $\sigma^{*}$ and $\sigma$ are cocycles, $\varphi\left(\sigma^{*}, \sigma\right)$ is a cocycle in $\mathbf{G}_{\mathbf{m}}\left(S^{(3)}\right)$. Indeed,

$$
\begin{aligned}
\Delta_{2} \varphi\left(\sigma^{*}, \sigma\right) & =\sigma_{1}^{*}\left(\sigma_{3}\right)_{1} \sigma_{1}^{*}\left(\sigma_{3}\right)_{2}^{-1} \sigma_{1}^{*}\left(\sigma_{3}\right)_{3} \sigma_{1}^{*}\left(\sigma_{3}\right)_{4}^{-1} \\
& =\sigma_{11}^{*}\left(\sigma_{31}\right) \sigma_{12}^{*}\left(-\sigma_{32}\right) \sigma_{13}^{*}\left(\sigma_{33}\right) \sigma_{14}^{*}\left(-\sigma_{34}\right) \\
& =\sigma_{11}^{*}\left(\sigma_{31}-\sigma_{32}\right)\left(\sigma_{13}^{*}-\sigma_{14}^{*}\right)\left(\sigma_{34}\right) \\
& =\sigma_{11}^{*}\left(\left(\sigma_{1}-\sigma_{2}\right)_{4}\right)\left(\left(\sigma_{2}^{*}-\sigma_{3}^{*}\right)_{1}\right)\left(\sigma_{34}\right) \\
& =\sigma_{11}^{*}\left(-\sigma_{34}\right) \sigma_{11}^{*}\left(\sigma_{34}\right)=1 .
\end{aligned}
$$

Also, if $\sigma$ or $\sigma^{*}$ is a coboundary, then $\varphi\left(\sigma^{*}, \sigma\right)$ is also a coboundary. For example, let $\sigma=\tau_{2}-\tau_{1}$ for some $\tau \in G(S)$, then

$$
\begin{aligned}
\Delta_{1}\left(\left(-\sigma^{*}\right)\left(\tau_{2}\right)\right) & =\left(\left(-\sigma^{*}\right)\left(\tau_{2}\right)\right)_{1}\left(\left(-\sigma^{*}\right)\left(\tau_{2}\right)\right)_{2}^{-1}\left(\left(-\sigma^{*}\right)\left(\tau_{2}\right)\right)_{3} \\
& =\left(-\sigma_{1}^{*}\right)\left(\tau_{21}\right)\left(\sigma_{2}^{*}\right)\left(\tau_{22}\right)\left(-\sigma_{3}^{*}\right)\left(\tau_{23}\right) \\
& =\left(-\sigma_{1}^{*}\right)\left(\tau_{13}\right)\left(\sigma_{2}^{*}-\sigma_{3}^{*}\right)\left(\tau_{23}\right) \\
& =\left(\sigma_{1}^{*}\right)\left(-\tau_{13}\right)\left(\sigma_{1}^{*}\right)\left(\tau_{23}\right) \\
& =\left(\sigma_{1}^{*}\right)\left(\left(\tau_{2}-\tau_{1}\right)_{3}\right)=\sigma_{1}^{*}\left(\sigma_{3}\right)=\varphi\left(\sigma^{*}, \sigma\right) .
\end{aligned}
$$

The first part of the lemma is proved. For the second part, observe that

$$
\begin{aligned}
H^{1}\left(R, G^{*}\right) \times H^{1}(R, G) & \cong \lim _{\longrightarrow} H^{1}\left(S / R, G^{*}\right) \times H^{1}(S / R, G) \\
& \stackrel{\varphi}{\longrightarrow} \stackrel{\lim _{\longrightarrow}}{\longrightarrow} H^{2}\left(S / R, \mathbf{G}_{\mathbf{m}}\right) \rightarrow H^{2}\left(R, \mathbf{G}_{\mathbf{m}}\right),
\end{aligned}
$$

where the limits are taken over all étale or flat coverings. 
2.2. Definition. We define dimodule versions of the groups $E_{1}, E_{2}$ introduced by Villamayor and Zelinsky in [36]. Let $S$ be a faithfully flat $R$-algebra, take $I \in \mathbf{P D}\left(S^{(n)}, G\right)$, and define $\delta_{n-1} I \in \mathbf{P D}\left(S^{(n+1)}, G\right)$ as follows:

$$
\delta_{n-1} I=I_{1} \otimes_{n-1} I_{2}^{*} \otimes_{n-1} \cdots \otimes_{n-1} I_{n+1}^{(*)} .
$$

Because $\delta_{n} \delta_{n-1}: S^{(n)} \rightarrow S^{(n+2)}$ is the zero map, it follows that we have a natural isomorphism of dimodules $\lambda_{I}: \delta_{n} \delta_{n-1} I \rightarrow S^{(n+2)}$.

Now, consider the category $\Omega$ with objects given by pairs $(I, \alpha)$, where $I \in \mathbf{P D}\left(S^{(n)}, G\right)$ and $\alpha: \delta_{n-1} I \rightarrow S^{(n+1)}$ is a dimodule isomorphism such that $\delta_{n} \alpha=\lambda_{I}$. A morphism between $(I, \alpha)$ and $(J, \beta)$ in $\Omega$ is given by an $S^{(n)}$ dimodule homomorphism $f: I \rightarrow J$ such that $\alpha=\beta \circ \delta_{n-1} f$. Consider the set $Z_{n}$ of isomorphism classes of $\Omega$. For $n=1, Z_{n}$ is made into a group by just taking the tensor product. For $n=2$, we consider the following, more complicated rule:

$$
(I, \alpha)(J, \beta)=\left(I \otimes_{2} J,(\alpha \otimes \beta) \varphi\left(e^{*}(I), e(J)\right)\right)
$$

where $e$ and $e^{*}$ are defined as in 1.4. Here our approach differs from the classical one by Villamayor and Zelinsky; as one may expect, the factor $\varphi\left(e^{*}(I), e(J)\right)$ is caused by the twisted multiplication in the smash product of two algebras, and this will become clear in the following proposition. Define $E_{1}=Z_{1}$ and $E_{2}=Z_{2} / B_{2}$, where $B_{2}$ is the subgroup of $Z_{2}$ consisting of elements of the form $\left(\delta_{0} J, \lambda_{J}\right)$. The fact that $B_{2}$ is a group follows from 2.1 .

2.3. Proposition. Suppose that $S$ is a faithfully flat $R$-algebra, and let $E_{1}, E_{2}$ be defined as above. Then $\operatorname{PD}(R, G)=E_{1}$, and there exists a monomorphism $i: \operatorname{BD}(S / R, G) \rightarrow E_{2}$, which is an isomorphism if $S$ is faithfully projective as an $R$-module.

Proof. The proof of the first statement is a straightforward application of 1.6 and 1.7. Let us give the proof of the second part in more detail. Take $[A] \in$ $\mathrm{BD}(S / R, G)$. Then we have a dimodule isomorphism $\rho: A \otimes S \rightarrow \operatorname{End}_{S}(Q)$, for some $Q \in \mathbf{F P D}(S, G)$. As usual in this kind of argument, define $\Phi$ by the commutativity of the following diagram:

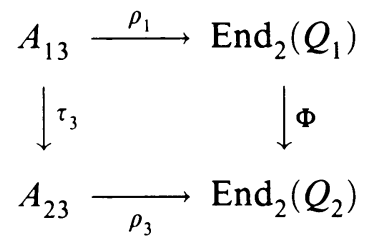

By 1.7, $\Phi$ is induced by a dimodule isomorphism $f: Q_{1} \otimes_{2} I \rightarrow Q_{2}$, for some $I \in \mathbf{P D}\left(S^{(2)}, G\right) . f_{2}^{-1} f_{3} f_{1}: Q_{11} \otimes_{3} I_{2}^{*} \otimes_{3} I_{3} \otimes_{3} I_{1} \rightarrow Q_{11}$ induces $\Phi_{2}^{-1} \Phi_{3} \Phi_{1}=$ 1 , so from the uniqueness in 1.7 , it follows that we have an $S^{(3)}$-dimodule isomorphism $u: S^{(3)} \rightarrow \delta_{1} I$. Consider the map

$$
Q_{11} \rightarrow Q_{11} \otimes_{3} S^{(3)} \stackrel{1 \otimes u}{\longrightarrow} Q_{11} \otimes_{3} \delta_{1} I \stackrel{f_{2}^{-1} f_{3} f_{1}}{\longrightarrow} Q_{11}
$$


which is given by multiplication by a unit $m \in \mathbf{G}_{\mathbf{m}}\left(S^{(3)}\right)$. It follows that the map

$$
f_{2}^{-1} f_{3} f_{1}: Q_{11} \otimes_{3} \delta_{1} I \rightarrow Q_{11}=Q_{11} \otimes_{3} S^{(3)}
$$

is of the form $1 \# m u^{-1}=1 \# m \alpha$. Define $i([A])=(I, \alpha)$. The proof of the fact that $i$ is a well-defined monomorphism is long but straightforward (after inspection of the proof by Villamayor and Zelinsky for the usual Brauer group). The most interesting point for us is to show that $i$ preserves multiplication, because here the special multiplication on $E_{2}$ comes into the picture. So let $\left[A^{\prime}\right]$ be another element of $\mathrm{BD}(S / R, G)$, and let $\rho^{\prime}, Q^{\prime}, \Phi^{\prime}, f^{\prime}, I^{\prime}$ be defined as above. Clearly $\Phi \# \Phi^{\prime}$ is induced by $f \otimes f^{\prime}: Q_{1} \otimes_{2} I \otimes_{2} Q_{1}^{\prime} \otimes_{2} I^{\prime} \rightarrow Q_{2} \otimes_{2} Q_{2}^{\prime}$. But if we want to calculate $\delta_{1}\left(f \# f^{\prime}\right)$, then we have the problem that we cannot interchange $I$ and $Q_{1}^{\prime}$. To overcome this, we observe that $\Phi$ and $\Phi^{\prime}$ are also induced by $\underline{f}: Q_{1} \otimes \underline{I} \rightarrow Q_{2}$ and $\underline{f}^{\prime}: Q^{\prime} \otimes \underline{I}^{\prime} \rightarrow Q_{2}^{\prime}$, where now $\underline{I}$ and $\underline{I}^{\prime}$ have no action or grading, but where $\underline{f}$ and $\underline{f}^{\prime}$ are not dimodule isomorphisms anymore. However, $\underline{f}$ and $\underline{f}^{\prime}$ are locally homogeneous of degree $\sigma$ and $\sigma^{\prime} \in G\left(S^{(2)}\right)$, and $G\left(S^{(2)}\right)$ acts on $\underline{f}$ and $\underline{f}^{\prime}$ by the following rules:

$$
\tau(\underline{f})=\sigma^{*}(\tau) \underline{f} \text { and } \tau\left(\underline{f}^{\prime}\right)=\sigma^{*}(\tau) \underline{f},
$$

where we denoted

$$
\left(e, e^{*}\right)(I)=\left(\sigma, \sigma^{*}\right) \text { and }\left(e, e^{*}\right)\left(I^{\prime}\right)=\left(\sigma^{\prime}, \sigma^{* \prime}\right) .
$$

Since $\underline{I}$ has trivial action and grading, it is no problem to interchange $\underline{I}$ and $Q_{1}^{\prime}$. Therefore, $\Phi \# \Phi^{\prime}$ is induced by $\underline{f} \# \underline{f}^{\prime}$, and

$$
\begin{aligned}
\Delta_{1}\left(\underline{f} \# \underline{f}^{\prime}\right) & =\left(\underline{f}_{2} \# \underline{f}_{2}^{\prime}\right)^{-1}\left(\underline{f}_{3} \# \underline{f}_{3}^{\prime}\right)\left(\underline{f}_{1} \# \underline{f}_{1}^{\prime}\right) \\
& =\sigma_{2}^{*}\left(\sigma_{2}^{\prime}\right) \sigma_{1}^{*}\left(\sigma_{3}^{\prime}\right)\left(\underline{f}_{2}^{-1} \# \underline{f}_{2}^{\prime-1}\right)\left(\underline{f}_{3} \underline{f}_{1} \# \underline{f}_{3}^{\prime} \underline{f}_{1}^{\prime}\right) \\
& =\sigma_{2}^{*}\left(\sigma_{2}^{\prime}\right) \sigma_{1}^{*}\left(\sigma_{3}^{\prime}\right)\left(\sigma_{3}^{*}+\sigma_{1}^{*}\right)\left(-\sigma_{2}^{\prime}\right)\left(\Delta_{1} \underline{f} \# \Delta_{1} \underline{f}^{\prime}\right) \\
& =\sigma_{1}^{*}\left(\sigma_{3}^{\prime}\right)\left(\Delta_{1} \underline{f} \# \Delta_{1} \underline{f}^{\prime}\right) .
\end{aligned}
$$

We have shown that $i\left(A \# A^{\prime}\right)=\left(I \#_{2} I^{\prime}, \varphi\left(e^{*}(I), e\left(I^{\prime}\right)\right)\left(\alpha \# \alpha^{\prime}\right)\right)$, so $i$ preserves multiplication. Let us show that $i$ is surjective if $S / R$ is faithfully projective. Take $(I, \alpha) \in E_{2}$.

$\alpha$ may be viewed as an $S^{(3)}$-dimodule isomorphism $\alpha: I_{1} \otimes_{3} I_{3} \rightarrow I_{2}$. Let $P$ be the $S$-dimodule which is equal to $I$ as a $\mathrm{Z}$-dimodule, but where $S$ acts "on the first factor", that is $s \cdot x=(s \otimes 1) x$, for $s \in S, x \in I$. Then $P$ is a faithfully projective $S$-module, and

$$
\begin{aligned}
& P_{1}=I_{1} \text {, with } S^{(2)} \text { acting "on the first two factors"; } \\
& P_{2}=I_{3} \text {, with } S^{(2)} \text { acting "on the first and third factor"; } \\
& P_{1} \otimes_{2} I=I_{1} \otimes_{3} I_{3} \text {, with } S^{(2)} \text { acting "on the first two factors". }
\end{aligned}
$$

Therefore, we obtain an isomorphism of $S^{(2)}$-dimodules

$$
f: P_{1} \otimes_{2} I_{2}=I_{1} \otimes_{3} I_{3} \stackrel{\alpha}{\longrightarrow} I_{2} \stackrel{\tau_{1}}{\longrightarrow} P_{2} .
$$


Note that $\alpha$ and $\tau_{1}$ are not $S^{(2)}$-dimodule isomorphisms, but their composition is an $S^{(2)}$-dimodule isomorphism. $f_{2}^{-1} f_{3} f_{1}$ is just multiplication by a unit, and therefore the induced map $\Psi: \operatorname{End}_{2}\left(P_{1}\right) \rightarrow \operatorname{End}_{2}\left(P_{2}\right)$ is a dimodule descent datum, descending to a $G$-Azumaya algebra $A$.

Consider the category of sheaves of abelian groups $S\left(R_{\mathrm{ff}}\right)$ on the flat site of $R$ (cf. [26]). Let $1 \rightarrow \mathbf{G}_{\mathbf{m}} \rightarrow F_{0} \rightarrow F_{1} \rightarrow \cdots$ be an injective resolution of $\mathbf{G}_{\mathbf{m}}$ in this category, and let $C_{q}=\operatorname{Ker}\left(F_{q} \rightarrow F_{q+1}\right)$ (thus $C_{0}=\mathbf{G}_{\mathbf{m}}$ ). For any faithfully flat $R$-algebra $S$, we have some exact sequences (cf. [36, 6.11]):

$$
\begin{array}{r}
1 \longrightarrow H^{1}\left(S / R, \mathbf{G}_{\mathbf{m}}\right) \stackrel{\alpha_{1}}{\longrightarrow} H^{1}\left(R, \mathbf{G}_{\mathbf{m}}\right) \stackrel{\beta_{1}}{\longrightarrow} H^{0}\left(S / R, H^{1}\left(\cdot, \mathbf{G}_{\mathbf{m}}\right)\right) \\
\stackrel{\gamma_{1}}{\longrightarrow} H^{2}\left(S / R, \mathbf{G}_{\mathbf{m}}\right) \stackrel{\alpha_{2}}{\longrightarrow} H^{1}\left(S / R, C_{1}\right) \stackrel{\beta_{2}}{\longrightarrow} H^{1}\left(S / R, H^{1}\left(\cdot, \mathbf{G}_{\mathbf{m}}\right)\right) \\
\stackrel{\gamma_{2}}{\longrightarrow} H^{3}\left(S / R, \mathbf{G}_{\mathbf{m}}\right) \stackrel{\alpha_{3}}{\longrightarrow} H^{2}\left(S / R, C_{1}\right) \stackrel{\beta_{3}}{\longrightarrow} \\
\cdots,
\end{array}
$$

$$
\begin{gathered}
1 \longrightarrow H^{1}\left(S / R, C_{1}\right) \longrightarrow H^{2}\left(R, \mathbf{G}_{\mathbf{m}}\right) \longrightarrow H^{0}\left(S / R, H^{2}\left(\cdot, \mathbf{G}_{\mathbf{m}}\right)\right) \\
\longrightarrow H^{2}\left(S / R, C_{1}\right) \longrightarrow H^{1}\left(S / R, C_{2}\right) \longrightarrow
\end{gathered}
$$

In the next proposition, the map $\alpha_{2}$ will be important. We will also consider the map

$$
\alpha_{2} \circ \varphi: H^{1}\left(S / R, G^{*}\right) \times H^{1}(S / R, G) \stackrel{\varphi}{\longrightarrow} H^{2}\left(S / R, \mathbf{G}_{\mathbf{m}}\right) \stackrel{\alpha_{2}}{\longrightarrow} H^{1}\left(S / R, C_{1}\right) .
$$

2.4. Proposition. Let $S / R$ be faithfully flat; then

$$
E_{2} \cong H^{1}\left(S / R, G \times G^{*}\right) \times_{\alpha_{2} \circ \varphi} H^{1}\left(S / R, C_{1}\right),
$$

where the multiplication on the product of cohomology groups is given by

$$
\left(\sigma, \sigma^{*}, c\right)\left(\sigma^{\prime}, \sigma^{\prime *}, c^{\prime}\right)=\left(\sigma+\sigma^{\prime}, \sigma^{*}+\sigma^{\prime *}, c c^{\prime} \alpha_{2}\left(\varphi\left(\sigma^{*}, \sigma^{\prime}\right)\right) .\right.
$$

Proof. We construct an isomorphism

$$
\delta: H^{1}\left(S / R, G \times G^{*}\right) \times_{\alpha_{2} \circ \varphi} H^{1}\left(S / R, C_{1}\right) \rightarrow E_{2} .
$$

Let $E_{2}^{\text {class }}$ be the classical analog of $E_{2}$, as introduced in [36]. It is clear that $E_{2}^{\text {class }}$ is a normal subgroup of $E_{2}$, and it is well known that we have an isomorphism $\underline{\delta}: H^{1}\left(S / R, C_{1}\right) \rightarrow E_{2}^{\text {class }}$. Also, for $u$ representing an element of $H^{2}\left(S / R, \mathbf{G}_{\mathbf{m}}\right)$, we have that $\underline{\delta}\left(\alpha_{2}(u)\right)=\left(S^{(2)}, m(u)\right)$, where $m(u)$ is given by multiplication by $u$.

If $\left(\sigma, \sigma^{*}\right)$ is a cocycle in $Z^{1}\left(S / R, G \times G^{*}\right)$, then the identity $i: \Delta_{1} S^{(2)}\left(\sigma, \sigma^{*}\right)$ $\rightarrow S^{(3)}$ is a dimodule isomorphism. Define

$$
\delta\left(\sigma, \sigma^{*}, c\right)=\underline{\delta}(c)\left(S^{(2)}\left(\sigma, \sigma^{*}\right), i\right) .
$$


Then

$$
\begin{aligned}
\delta((\sigma, & \left.\left.\sigma^{*}, c\right)\left(\sigma^{\prime}, \sigma^{\prime *}, c^{\prime}\right)\right) \\
& =\delta\left(\sigma+\sigma^{\prime}, \sigma^{*}+\sigma^{\prime *}, c c^{\prime} \alpha_{2}\left(\varphi\left(\sigma^{*}, \sigma^{\prime}\right)\right)\right) \\
& =\underline{\delta}(c) \underline{\delta}\left(c^{\prime}\right)\left(S^{(2)}, m\left(\varphi\left(\sigma^{*}, \sigma^{\prime}\right)\right)\right)\left(S^{(2)}\left(\sigma+\sigma^{\prime}, \sigma^{*}+\sigma^{\prime *}\right), i\right) \\
& =\underline{\delta}(c) \underline{\delta}\left(c^{\prime}\right)\left(S^{(2)}\left(\sigma, \sigma^{*}\right), i\right)\left(S^{(2)}\left(\sigma^{\prime}, \sigma^{\prime *}\right), i\right) \\
& =\delta\left(\sigma, \sigma^{*}, c\right) \delta\left(\sigma^{\prime}, \sigma^{\prime *}, c^{\prime}\right)
\end{aligned}
$$

so $\delta$ is a homomorphism. The fact that $\delta$ is an isomorphism follows easily now from the fact that $\underline{\delta}$ is an isomorphism.

2.5. Corollary. We have a monomorphism

$$
\theta: \operatorname{BD}^{s}(R, G) \rightarrow H^{1}\left(R_{\mathrm{fl}}, G \times G^{*}\right) \times{ }_{\varphi} H^{2}\left(R, \mathbf{G}_{\mathbf{m}}\right) .
$$

Proof. For any faithfully flat $R$-algebra $S$, we have, by 2.3, 2.4 and (2.3.2):

$$
\begin{aligned}
\mathrm{BD}(S / R) & \subset E_{2}=H^{1}\left(S / R, G \times G^{*}\right) \times{ }_{\alpha_{2} \circ \varphi} H^{1}\left(S / R, C_{1}\right) \\
& \rightarrow H^{1}\left(R_{\mathrm{fl}}, G \times G^{*}\right) \times{ }_{\varphi} H^{2}\left(R, \mathbf{G}_{\mathbf{m}}\right) .
\end{aligned}
$$

We obtain the result if we take the union over all faithfully flat $S$.

2.6. Corollary (Chase-Rosenberg exact sequences). Let $S$ be a faithfully projective $R$-algebra; then we have exact sequences

$$
\begin{aligned}
1 & \rightarrow\left(G \times G^{*}\right)(R) \times H^{1}\left(S / R, \mathbf{G}_{\mathbf{m}}\right) \rightarrow \mathrm{PD}(R, G) \rightarrow H^{0}(S / R, \mathrm{Pic}) \\
& \rightarrow H^{1}\left(S / R, G \times G^{*}\right) \times{ }_{\varphi} H^{2}\left(S / R, \mathbf{G}_{\mathbf{m}}\right) \rightarrow \mathrm{BD}(S / R, G) \rightarrow H^{1}(S / R, \mathrm{Pic})
\end{aligned}
$$

$$
\begin{aligned}
1 & \rightarrow H^{1}\left(S / R, \mathbf{G}_{\mathbf{m}}\right) \rightarrow \mathrm{PD}(R, G) \rightarrow H^{0}(S / R, \mathrm{PD}(\cdot, G)) \\
& \rightarrow H^{2}\left(S / R, \mathbf{G}_{\mathbf{m}}\right) \rightarrow \mathrm{BD}(S / R, G) \rightarrow H^{1}(S / R, \mathrm{PD}(\cdot, G)) \rightarrow H^{3}\left(S / R, \mathbf{G}_{\mathbf{m}}\right) .
\end{aligned}
$$

Proof. (2.6.1) follows from (2.3.1) and the results above. (2.6.2) cannot be derived from (2.2.1), and the most elementary way to prove it is to adapt Knus' proof [21] of the classical Chase-Rosenberg sequence [10]. We leave the details of this to the reader.

2.7. Theorem. $\operatorname{BD}^{s}(R, G) \cong H^{1}\left(R_{\mathrm{fl}}, G \times G^{*}\right) \times{ }_{\varphi} H^{2}\left(R, \mathbf{G}_{\mathbf{m}}\right)_{\text {tors }}$.

Proof. From Gabber's Theorem (cf. [18]), $H^{2}\left(R_{\mathrm{fl}}, \mathbf{G}_{\mathrm{m}}\right)_{\mathrm{tors}} \cong H^{2}\left(R_{\mathrm{et}}, \mathbf{G}_{\mathrm{m}}\right)_{\mathrm{tors}} \cong$ $\operatorname{Br}(R)$, a normal subgroup of $\operatorname{BD}^{s}(R, G)$. So it remains to show that $\operatorname{BD}^{s}(R, G)$ $\rightarrow H^{1}\left(R_{\mathrm{fl}}, G \times G^{*}\right)$ is surjective. This follows from the propositions above and the fact that every element of $H^{1}\left(R_{\mathrm{fl}}, G \times G^{*}\right)$ may be represented by an element of $H^{1}\left(S / R, G \times G^{*}\right)$ for some faithfully projective algebra $S$ (cf. 1.13). This is the only point in the description of the Brauer-Long group where we need Galois theory.

2.8. Note. If $G(R) \cong G^{* *}(R)$, and if the order of $G$ is invertible in $R$, then the flat site in the above theorem may be replaced by the etale site; this will 
also follow from the results in $\S 4$. In this case, the map $\theta$ can be described in an elementary way: take $[A] \in \mathrm{BD}(S / R, G)$, for some étale covering $S$ of $R$. Then we have an isomorphism $\psi: A \otimes S \rightarrow \operatorname{End}_{S}(P)$, for some $P \in$ $\operatorname{FPD}(S, G)$, defining a commutative diagram

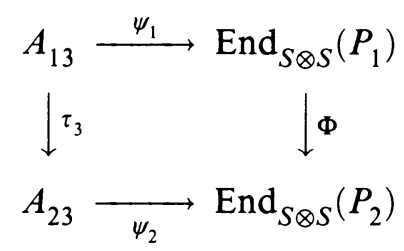

By 1.7, $\Phi$ is induced by some $f: P_{1} \otimes_{2} I \rightarrow P_{2}$ for some $I \in \mathbf{P D}\left(S^{(2)}, G\right)$. Invoking Artin's refinement theorem [1], we can replace $S$ by another étale covering such that $[I]=1$ in $\operatorname{Pic}\left(S^{(2)}\right)$, but not in $\operatorname{PD}\left(S^{(2)}, G\right)$. Thus $I \cong_{d}$ $S^{(2)}\left(\sigma, \sigma^{*}\right)$, for some $\left(\sigma, \sigma^{*}\right) \in\left(G \times G^{*}\right)\left(S^{(2)}\right)$. It is well known that $f_{2}^{-1} f_{3} f_{1} \in$ $Z^{2}\left(S / R, \mathbf{G}_{\mathrm{m}}\right)$, and it is easy to see that $\left(\sigma, \sigma^{*}\right) \in Z^{1}\left(S / R, G \times G^{*}\right)$, since $\Phi$ is a dimodule isomorphism. This defines the map $\theta$.

\section{AN EXACT SEQUENCE DESCRIBING THE COMPLETE BRAUER-LONG GROUP}

Let $A$ be an $R$ - $G$-dimodule algebra; the group of $R$ - $G$-dimodule automorphisms of $A$ is denoted by $G$-Aut $R_{R}(A)$. Following [5], an element $f$ of $G$-Aut ${ }_{R}(A)$ is called $G$-inner if there exists $u \in \mathbf{G}_{\mathbf{m}}(A)$ such that $f(a)=$ $\alpha(u) a u^{-1}$, for all $a$ homogeneous of degree $\alpha$. In [5, Proposition 1.2], the second author gave a dimodule version of an exact sequence due to Rosenberg and Zelinsky [30], generalizing the Skolem-Noether theorem. If $A$ is a $G-R$ Azumaya algebra, then the following sequence is exact:

$$
1 \rightarrow G-\operatorname{Inn}_{R}(A) \rightarrow G-\operatorname{Aut}_{R}(A) \rightarrow \operatorname{Pic}(R),
$$

where $G$-Inn $\operatorname{In}_{R}(A)$ represents the subgroup of $G$-inner automorphisms of $G$ $\operatorname{Aut}_{R}(A)$. The image of $f \in G-\mathrm{Aut}_{R}(A)$ is represented by $I_{f}=\{x \in A$ : $\alpha(x) a=f(a) x$, for all $\left.\alpha \in G, a \in A_{\alpha}\right\}$. In the sequel, we will need another dimodule variant of the Rosenberg-Zelinsky sequence. Let $G-\mathrm{INN}_{R}(A)$ be the subgroup of $G$ - $\operatorname{Inn}_{R}(A)$ of automorphisms induced by an element $u$ of $A$ which is homogeneous of degree 0 and on which $G$ acts trivially.

3.1. Theorem. Let $A$ be an $R$-G-Azumaya algebra. We have an exact sequence

$$
1 \rightarrow G-\mathrm{INN}_{R}(A) \rightarrow G-\mathrm{Aut}_{R}(A) \rightarrow \mathrm{PD}(R, G) .
$$

Proof. For $f \in G$-Aut ${ }_{R}(A)$, we define $I_{f}$ as above. From [5], it follows that $I_{f}$ is an invertible module, and it is clearly a subdimodule of $A$. Define $\Phi_{A}(f)=\left[I_{f}\right] \in \operatorname{PD}(R, G)$. Suppose that $\left[\Phi_{A}(f)\right]=1$. Then $I_{f}=R u$, for some $u \in A_{0}^{G}$. From the definition of $I_{f}$, it follows that $f(a)=u a u^{-1}$, so $f \in G-\mathrm{INN}_{R}(A)$.

Recall from $\S 1$ that the $G$-dimodule structure on $A$ defines a $\left(G \times G^{*}\right)(R)$ module structure on $A$. So we have a map $\left(m, m^{*}\right):\left(G \times G^{*}\right)(R) \rightarrow G-\operatorname{Aut}_{R}(A)$. 
Define $\alpha_{A} \in \operatorname{End}\left(G \times G^{*}\right)(R)$ to be the composition $\alpha_{A}=\left(e, e^{*}\right) \circ \Phi_{A} \circ\left(m, m^{*}\right)$, where $\left(e, e^{*}\right)$ is as in Lemma 1.4. $\beta_{A}$ is defined to be $I-\alpha_{A}$, where $I$ is the identity map on $\left(G \times G^{*}\right)(R)$ (recall that $G$ and $G^{*}$ are denoted additively).

3.2. Theorem. $\beta_{A}$ defines a group homomorphism

$$
\beta: \mathrm{BD}(R, G) \rightarrow \operatorname{Aut}\left(G \times G^{*}\right)(R),
$$

given by $\beta([A])=\beta_{A}$.

Notes. (1) In Theorem 3.2, $\operatorname{Aut}\left(G \times G^{*}\right)(\cdot)$ is the sheaf associated to the constant presheaf $\operatorname{Aut}\left(G \times G^{*}\right)$. Thus $\operatorname{Aut}\left(G \times G^{*}\right)(R)$ is the group of continuous (that is locally constant) maps from $\operatorname{Spec}(R)$ to $\operatorname{Aut}\left(G \times G^{*}\right)$.

(2) $\beta$ generalizes maps defined by Orzech in [28], and by Childs in [12]. Orzech's map is defined only on the subset $\operatorname{BAz}(R, G)$ of $\operatorname{BD}(R, G)$ represented by central separable $G$-Azumaya algebras, in the case where this subset forms a group. If $G(R) \cong G^{* *}(R)$ and $|G|^{-1} \in R$, this comes down to restricting to $G$ a cyclic group. Orzech then defines a map $\operatorname{BAz}(R, G) \rightarrow \operatorname{Aut}(G(R))$. In our description $\operatorname{Aut}(G(R))$ embeds in $\operatorname{Aut}\left(G \times G^{*}\right)(R)$ by $f \rightarrow\left(f, f^{*-1}\right)$ (cf. 3.7).

To define Childs' map, one has to restrict to products of $p$-groups, each of which is the product of a cyclic group of the same order, and to the case where $G(\cdot) \cong G^{* *}(\cdot),|G|$ invertible. Childs' map is defined on the whole Brauer-Long group, but is an antihomomorphism, corresponding to our map after every element is sent to its inverse. Some of the technical difficulties that Childs and Orzech encounter defining their maps are avoided or simplified in our approach.

Proof.

$$
\beta_{A \# B}=\beta_{A} \circ \beta_{B} .
$$

We have to show that $\alpha_{A \# B}=\alpha_{A} \circ \beta_{B}+\alpha_{B}$. Let $\alpha_{B}\left(\sigma, \sigma^{*}\right)=\left(\tau, \tau^{*}\right)$, $\beta_{B}\left(\sigma, \sigma^{*}\right)=\left(\sigma-\tau, \sigma^{*}-\tau^{*}\right)=\left(\rho, \rho^{*}\right), \alpha_{A}\left(\beta_{B}\left(\sigma, \sigma^{*}\right)\right)=\left(\gamma, \gamma^{*}\right)$. Take $u \in \Phi_{A}\left(\rho, \rho^{*}\right), v \in \Phi_{B}\left(\sigma, \sigma^{*}\right)$, then $\alpha(u)=\gamma^{*}(\alpha) u$ and $\alpha(v)=\tau^{*}(\alpha) v$, for all $\alpha \in G(R)$. For all $a \in A_{\alpha}, b \in B_{\beta}$, we have

$$
\begin{aligned}
\left(\left(\sigma, \sigma^{*}\right)(a \# b)\right)(u \# v) & =\left(\left(\sigma, \sigma^{*}\right)(a) \#\left(\sigma, \sigma^{*}\right)(b)\right)(u \# v) \\
& =\left(\sigma, \sigma^{*}\right)(a) \beta(u) \#\left(\sigma, \sigma^{*}\right)(b) v \\
& =(\alpha \beta)(u)\left(\tau, \tau^{*}\right)(a) \# \beta(v) b \\
& =(\alpha \beta)(u) \tau^{*}(\alpha) \tau(a) \# \beta(v) b \\
& =\left((\alpha \beta)(u) \# \tau^{*}(\alpha) \beta(v)\right)(a \# b) \\
& =((\alpha \beta)(u \# v))(a \# b) .
\end{aligned}
$$

Hence $u \# v \in \Phi_{A \# B}\left(\sigma, \sigma^{*}\right)$, and $\alpha_{A \# B}\left(\sigma, \sigma^{*}\right)=\left(\tau+\gamma, \tau^{*}+\gamma^{*}\right)=\alpha_{A}\left(\beta_{B}\left(\sigma, \sigma^{*}\right)\right)$ $+\alpha_{B}\left(\sigma, \sigma^{*}\right)$.

$$
\text { If } A=\operatorname{End}_{R}(P), \text { then } \alpha_{A}=0 \text { and } \beta_{A}=I \text {. }
$$


Indeed, the automorphism $P \rightarrow P: x \rightarrow\left(\sigma, \sigma^{*}\right)(x)$ is multiplication by an element of $\left(A_{0}\right)^{G}$, and induces the action of $\left(\sigma, \sigma^{*}\right) \in\left(G \times G^{*}\right)(R)$ on $A=$ $\operatorname{End}_{R}(P)$. Hence this action is dimodule inner, and the result follows.

$$
\beta_{A} \in \operatorname{Aut}\left(G \times G^{*}\right)(R) .
$$

From (3.2.1) and (3.2.2), it follows that $\beta_{A} \circ \beta_{\bar{A}}=\beta_{\bar{A}} \circ \beta_{A}=I$, so $\beta_{A}$ is invertible, and therefore an automorphism.

Our next aim is to describe the kernel and image of the map $\beta$. We will consider the canonical map $l: G(R) \rightarrow G^{* *}(R)$. Define the map $t$ as follows:

$$
t:\left(G \times G^{*}\right)(R) \stackrel{\text { switch }}{\longrightarrow}\left(G^{*} \times G\right)(R) \stackrel{1 \times l}{\longrightarrow}\left(G^{*} \times G^{* *}\right)(R) \cong\left(G \times G^{*}\right)^{*}(R) .
$$

Hence $t\left(\sigma, \sigma^{*}\right)=\left(\sigma^{*}, l(\sigma)\right)$. We also define $\Phi:\left(G \times G^{*}\right)(R) \rightarrow\left(G \times G^{*}\right)^{*}(R)$ by $\Phi\left(\sigma, \sigma^{*}\right)=\left(\sigma^{*}, 0\right)$. Clearly the dual map

$$
\Phi^{*}:\left(G^{* *} \times G^{* * *}\right)(R) \rightarrow\left(G \times G^{*}\right)^{*}(R)
$$

is given by $\Phi^{*}\left(\sigma^{* *}, \sigma^{* * *}\right)=\left(0, \sigma^{* *}\right)$. Therefore $t=\Phi+\Phi^{*} l$, where now $l:\left(G \times G^{*}\right)(R) \rightarrow\left(G^{* *} \times G^{* * *}\right)(R)$.

For $\sigma \subset G, \sigma^{*} \in G^{*}$, we will often use the duality notation $\left\langle\sigma, \sigma^{*}\right\rangle=$ $\left\langle\sigma^{*}, \sigma\right\rangle=\sigma^{*}(\sigma)$. On $\left(G \times G^{*}\right)(R)$, we define a quadratic form $q$ and a bilinear form $b$ in the following way: $q\left(\sigma, \sigma^{*}\right)=\left\langle\left(\sigma, \sigma^{*}\right), \Phi\left(\sigma, \sigma^{*}\right)\right\rangle=\sigma^{*}(\sigma)$ and $b\left(\left(\sigma, \sigma^{*}\right),\left(\tau, \tau^{*}\right)\right)=\left\langle\left(\sigma, \sigma^{*}\right), t\left(\tau, \tau^{*}\right)\right\rangle=\sigma^{*}(\tau) \tau^{*}(\sigma)$. We define

$$
\begin{aligned}
O(R, G)_{\min } & =\left\{f \in \operatorname{Aut}\left(G \times G^{*}\right)(R): q \circ f=q\right\} \\
O(R, G)_{\max } & =\left\{f \in \operatorname{Aut}\left(G \times G^{*}\right)(R): b \circ(f, f)=b\right\} \\
& =\left\{f \in \operatorname{Aut}\left(G \times G^{*}\right)(R): f^{*} \circ t \circ f=t\right\} .
\end{aligned}
$$

3.3. Proposition. $\operatorname{Im} \beta \subset O(R, G)_{\min } \subset O(R, G)_{\max }$; if 2 does not divide the order of $G$, then $O(R, G)_{\min }=O(R, G)_{\max }$.

Proof. First, take $f \in \operatorname{Im} \beta$, hence $f=\beta_{A}, I-f=\alpha_{A}$ for some $G$-Azumaya algebra $A$. Take $\left(\sigma, \sigma^{*}\right) \in\left(G \times G^{*}\right)(R)$, and let $\alpha_{A}\left(\sigma, \sigma^{*}\right)=\left(\tau, \tau^{*}\right)$. Let $x$ be an arbitrary element of $\Phi_{A}\left(\sigma, \sigma^{*}\right)$ so that $\operatorname{deg} x=\tau$ and $\rho(x)=\tau^{*}(\rho)$ for all $\rho \in G(R)$. Let $a$ be an element of $A$ of degree $\alpha$. From the definition of $\Phi_{A}\left(\sigma, \sigma^{*}\right)$, it follows that

$$
\alpha(x) a=\sigma^{*}(\alpha) \sigma(a) x .
$$

Taking $a=x, \alpha=\tau$, it follows that for all $x \in \Phi_{A}\left(\sigma, \sigma^{*}\right)$ :

$$
\tau^{*}(\tau) x^{2}=\sigma^{*}(\tau) \tau^{*}(\sigma) x^{2},
$$


and therefore

$$
\begin{aligned}
& \tau^{*}(\tau)=\sigma^{*}(\tau) \tau^{*}(\sigma), \quad \text { or } \\
& \left\langle\left(\tau, \tau^{*}\right), \Phi\left(\tau, \tau^{*}\right)\right\rangle=\left\langle\left(\tau, \tau^{*}\right), t\left(\sigma, \sigma^{*}\right)\right\rangle, \quad \text { or } \\
& \left\langle\alpha_{A}\left(\sigma, \sigma^{*}\right),\left(\Phi \alpha_{A}-t\right)\left(\sigma, \sigma^{*}\right)\right\rangle=1, \quad \text { or } \\
& \left\langle(I-f)\left(\sigma, \sigma^{*}\right),\left(\Phi f+\Phi^{*}{ }^{\prime}\right)\left(\sigma, \sigma^{*}\right)\right\rangle=1, \quad \text { or } \\
& \left\langle\left(\sigma, \sigma^{*}\right), \Phi f\left(\sigma, \sigma^{*}\right)\right\rangle\left\langle\left(\sigma, \sigma^{*}\right), \Phi^{*} i\left(\sigma, \sigma^{*}\right)\right\rangle \\
& \quad=\left\langle f\left(\sigma, \sigma^{*}\right), \Phi f\left(\sigma, \sigma^{*}\right)\right\rangle\left\langle f\left(\sigma, \sigma^{*}\right), \Phi^{*} i\left(\sigma, \sigma^{*}\right)\right\rangle, \quad \text { or } \\
& \left\langle\left(\sigma, \sigma^{*}\right), \Phi\left(\sigma, \sigma^{*}\right)\right\rangle=\left\langle f\left(\sigma, \sigma^{*}\right), \Phi f\left(\sigma, \sigma^{*}\right)\right\rangle .
\end{aligned}
$$

It follows that $f \in O(R, G)_{\min }$. Next, take $f \in O(R, G)_{\min }$. For all $\left(\sigma, \sigma^{*}\right)$, $\left(\tau, \tau^{*}\right) \in\left(G \times G^{*}\right)(R)$, we have:

$$
\begin{aligned}
& \left\langle\left(\sigma, \sigma^{*}\right)+\left(\tau, \tau^{*}\right), \Phi\left(\sigma, \sigma^{*}\right)+\Phi\left(\tau, \tau^{*}\right)\right\rangle \\
& =\left\langle f\left(\sigma, \sigma^{*}\right)+f\left(\tau, \tau^{*}\right), \Phi f\left(\sigma, \sigma^{*}\right)+\Phi f\left(\tau, \tau^{*}\right)\right\rangle, \quad \text { or } \\
& \left\langle\left(\sigma, \sigma^{*}\right), \phi\left(\tau, \tau^{*}\right)\right\rangle\left\langle\left(\tau, \tau^{*}\right), \phi\left(\sigma, \sigma^{*}\right)\right\rangle \\
& =\left\langle f\left(\sigma, \sigma^{*}\right), \phi f\left(\tau, \tau^{*}\right)\right\rangle\left\langle f\left(\tau, \tau^{*}\right), \phi f\left(\sigma, \sigma^{*}\right)\right\rangle, \quad \text { or } \\
& \left\langle\left(\sigma, \sigma^{*}\right), t\left(\tau, \tau^{*}\right)\right\rangle=\left\langle f\left(\sigma, \sigma^{*}\right), t f\left(\tau, \tau^{*}\right)\right\rangle .
\end{aligned}
$$

So $f \in O(R, G)_{\max }$. Finally, suppose that 2 does not divide the order of $G$. Then $f$ satisfies (3.3.3), and therefore it satisfies (3.3.2). Taking $\left(\sigma, \sigma^{*}\right)=$ $\left(\tau, \tau^{*}\right)$, we obtain that $u^{2}=1$, where

$$
u=\left\langle\left(\sigma, \sigma^{*}\right), \Phi\left(\sigma, \sigma^{*}\right)\right\rangle\left\langle f\left(\sigma, \sigma^{*}\right), \Phi f\left(\sigma, \sigma^{*}\right)\right\rangle^{-1} .
$$

Now $u$ is also a $|G|$ th root of unity, so $u=1$, because $(|G|, 2)=1$. So $f \in O(R, G)_{\min }$.

We can now state the main theorem of this paper:

3.4. Theorem. Suppose that $|G|$ is invertible in $R$, and that $G(R)$ and $G^{* *}(R)$ are canonically isomorphic. Then $\operatorname{BD}(R, G)$ is described by the following exact sequence:

$$
1 \rightarrow \mathrm{BD}^{s}(R, G) \rightarrow \mathrm{BD}(R, G) \stackrel{\beta}{\longrightarrow} O(R, G)_{\min } \rightarrow 1 .
$$

Proof. First, we have to show that $\operatorname{Ker} \beta=\mathrm{BD}^{s}(R, G)$.

(3.4.1) Let $S$ be a faithfully flat $R$-algebra. We have the following commutative diagram of group homomorphisms:

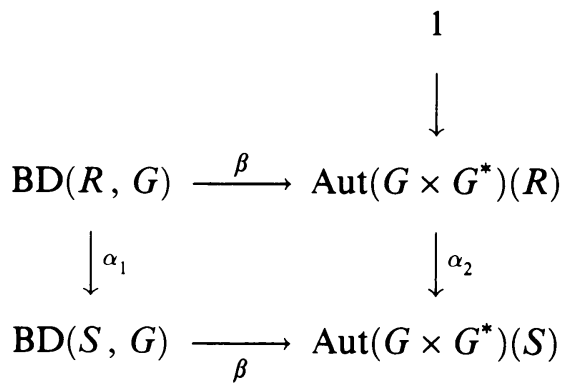


Because $\alpha_{2}$ is injective, it follows that $\operatorname{BD}(S / R, G)=\operatorname{Ker} \alpha_{1}$ is contained in $\operatorname{Ker} \beta$.

(3.4.2) Suppose that $\beta([A])=1$. Take a faithfully flat $R$-algebra $S$ such that the invertible $G$-dimodules $\Phi_{A \otimes S}\left(\sigma, \sigma^{*}\right)$ are free for all $\left(\sigma, \sigma^{*}\right) \in\left(G \times G^{*}\right)(S)$ (i.e. they represent trivial elements of $\operatorname{Pic}(S)$ but not of $\operatorname{PD}(S, G)) . Z(A \otimes S)$ is an abelian extension of $S$. Take $\sigma \in G$, then $\Phi_{A \otimes S}(\sigma, 0)=S u$ for some invertible $u$ in $A_{0} \cap A^{G}$, because $\alpha_{A}(\sigma, 0)=(0,0)$. Thus for all $a \in A \otimes S$, $\sigma(a)=u a u^{-1}$, and $\sigma(a)=a$ if $a \in Z(A \otimes S)$, and $A \otimes S$ is $S$-central. Replace $S$ by another faithfully flat extension $S$ such that $A \otimes S=\operatorname{End}_{S}(P)$ for some faithfully projective $S$-module $P$. Let $G=\left\langle\sigma_{1}\right\rangle \times \cdots \times\left\langle\sigma_{n}\right\rangle$, take one of its generators $\sigma$, and suppose that $m \sigma=0$. If the action of $\sigma$ is induced by $u$, then $u^{m}$ is a unit in $S$. Replacing $S$ by another faithfully flat extension, we may assume that $u^{m}=1$. We define an action of $\langle\sigma\rangle$ on $P$ by $\sigma(x)=u(x)$. Doing this for the $n$ generators of $G$, we obtain an action of $G$ on $P$, and in a similar way, we can define a $G^{*}$-action on $P$. Clearly the $G \times G^{*}$-action on $A \otimes S$ is induced by that on $P$; we therefore have a $G$-dimodule structure on $P$ inducing that on $A \otimes S=\operatorname{End}_{S}(P)$. Thus $[A \otimes S]=1$ in $\operatorname{BD}(S, G)$.

(3.4.3) Let us now show that $\beta$ is surjective. From now on, we will identify $G(R)$ and $G^{* *}(R)$, and therefore omit the $l: G(R) \rightarrow G^{* *}(R)$. Then the map $t$ introduced after 3.2 is invertible, and $t=\Phi+\Phi^{*}$. Therefore $t=t^{*}$. Take $f \in$ $O(R, G)_{\min }$. Let us first assume that $f$ is constant on $\operatorname{Spec}(R)$ (cf. the remark following Theorem 3.2). Let $a=(I-f) t^{-1}:\left(G^{*} \times G\right)(R) \rightarrow\left(G \times G^{*}\right)(R)$, then $a=t^{-1}\left(I-\left(f^{*}\right)^{-1}\right)$, because $t=f^{*} t f$, and $a^{*}=\left(I-f^{-1}\right) t^{-1}=t^{-1}\left(I-f^{*}\right)$. Also $f=I-a t, f^{*}=I-t a^{*}, f^{-1}=I-a^{*} t,\left(f^{*}\right)^{-1}=I-t a$. From the fact that $t=f^{*} t f$, it then follows that $t^{-1}=f^{-1} t^{-1}\left(f^{*}\right)^{-1}=\left(I-a^{*} t\right) t^{-1}(I-t a)=$ $t^{-1}-a^{*}-a+a^{*} t a$. Thus $a+a^{*}-a^{*} t a=0$. We define $d=a^{*} \Phi a-a^{*}$. It then follows that $d+d^{*}=0$.

Also, from the definition of $O(R, G)_{\min }$, we know that for all $\left(\sigma, \sigma^{*}\right) \in$ $\left(G \times G^{*}\right)(R):\left\langle\left(\sigma, \sigma^{*}\right),\left(\Phi-f^{*} \Phi f\right)\left(\sigma, \sigma^{*}\right)\right\rangle=1$. Now $\Phi-f^{*} \Phi f=\Phi-$ $\left(I-t a^{*}\right) \Phi(I-a t)=t a^{*} \Phi+\Phi a t-t a^{*} \Phi a t$. Hence:

$$
\begin{aligned}
1 & =\left\langle\left(\sigma, \sigma^{*}\right), t a^{*} \Phi\left(\sigma, \sigma^{*}\right)\right\rangle\left\langle\left(\sigma, \sigma^{*}\right),\left(\Phi a t-t a^{*} \Phi a t\right)\left(\sigma, \sigma^{*}\right)\right\rangle \\
& =\left\langle\Phi^{*} a\left(\sigma^{*}, \sigma\right),\left(\sigma, \sigma^{*}\right)\right\rangle\left\langle\left(\sigma, \sigma^{*}\right),\left(\Phi a-t a^{*} \Phi a\right)\left(\sigma^{*}, \sigma\right)\right\rangle \\
& =\left\langle\left(\sigma^{*}, \sigma\right), d\left(\sigma^{*}, \sigma\right)\right\rangle .
\end{aligned}
$$

(3.4.4) We claim that $\operatorname{Ker} a=\operatorname{Ker} a^{*}$. Indeed, the following statements are equivalent:

$$
\begin{gathered}
a\left(\sigma^{*}, \sigma\right)=0 ; \quad t^{-1}\left(I-\left(f^{*}\right)^{-1}\right)\left(\sigma^{*}, \sigma\right)=0 ; \quad\left(\sigma^{*}, \sigma\right)=\left(f^{*}\right)^{-1}\left(\sigma^{*}, \sigma\right) \\
f^{*}\left(\sigma^{*}, \sigma\right)=\left(\sigma^{*}, \sigma\right) ; \quad t^{-1}\left(I-f^{*}\right)\left(\sigma^{*}, \sigma\right)=0 ; \quad a^{*}\left(\sigma^{*}, \sigma\right)=0 .
\end{gathered}
$$

(3.4.5) On the $R$-module $A=R\left(\left(G^{*} \times G\right) / \operatorname{Ker} a\right)$, we define a multiplication 
as follows: fix a set of generators $\left\{e_{1}, \ldots, e_{n}\right\}$ of $G^{*} \times G$, and let

$$
\begin{gathered}
u_{\sum n_{i} e_{i}}=\prod u_{e_{i}}^{n_{i}}, \\
u_{\left(\sigma^{*}, \sigma\right)} u_{\left(\tau^{*}, \tau\right)}=\left\langle\left(\sigma^{*}, \sigma\right), d\left(\tau^{*}, \tau\right)\right\rangle u_{\left(\tau^{*}, \tau\right)} u_{\left(\sigma^{*}, \sigma\right)} .
\end{gathered}
$$

For less cumbersome notation, we write $u_{\left(\sigma^{*}, \sigma\right)}$ instead of $u_{\left[\left(\sigma^{*}, \sigma\right)\right]}$. Because of (3.4.3), and because $\operatorname{Ker} a$ is contained in $\operatorname{Ker} d=\operatorname{Ker}\left(a^{*} \Phi a-a^{*}\right)$, by (3.4.4), we have a well-defined multiplication. On $A$ we define a $G \times G^{*}$-grading as follows: $\operatorname{deg}_{G \times G^{*}} u_{\left(\sigma^{*}, \sigma\right)}=a\left(\sigma^{*}, \sigma\right)$. This defines a $G$-dimodule structure on $A: \operatorname{deg}_{G} u_{\left(\sigma^{*}, \sigma\right)}=p_{1} a\left(\sigma^{*}, \sigma\right)$ and $\tau\left(u_{\left(\sigma^{*}, \sigma\right)}\right)=\left\langle(\tau, 0), t a\left(\sigma^{*}, \sigma\right)\right\rangle=$ $\left\langle\left(\tau, \tau^{*}\right), \Phi a\left(\sigma^{*}, \sigma\right)\right\rangle$, where $p_{1}: G \times G^{*} \rightarrow G$ is just projection on the first factor and $\tau^{*}$ is arbitrary.

(3.4.6) $A$ is $R$-G-central. We have to show that

$\{x \in A: s x=\sigma(x) s$, for all homogeneous $s \in A$ of degree $\sigma\}=R$;

$\{x \in A: x$ homogeneous of degree $\xi, x s=\xi(s) x$ for all $s \in A\}=R$.

Indeed, suppose that for all $\left(\sigma^{*}, \sigma\right) \in G^{*} \times G$;

$$
u_{\left(\sigma^{*}, \sigma\right)} u_{\left(\tau^{*}, \tau\right)}=p_{1} a\left(\sigma^{*}, \sigma\right) u_{\left(\tau^{*}, \tau\right)} u_{\left(\sigma^{*}, \sigma\right)} .
$$

The right-hand side is equal to

$$
\left\langle a\left(\sigma^{*}, \sigma\right), \Phi a\left(\tau^{*}, \tau\right)\right\rangle\left\langle\left(\tau^{*}, \tau\right), d\left(\sigma^{*}, \sigma\right)\right\rangle u_{\left(\sigma^{*}, \sigma\right)} u_{\left(\tau^{*}, \tau\right)} .
$$

Therefore, we have for all $\left(\sigma^{*}, \sigma\right):\left\langle\left(\sigma^{*}, \sigma\right),\left(a^{*} \Phi a+d^{*}\right)\left(\tau^{*}, \tau\right)\right\rangle=1$, so $0=\left(a^{*} \Phi a+d^{*}\right)\left(\tau^{*}, \tau\right)=\left(a^{*} \Phi a-d\right)\left(\tau^{*}, \tau\right)=a^{*}\left(\tau^{*}, \tau\right)$, and $\left(\tau^{*}, \tau\right) \in \operatorname{Ker} a^{*}=$ $\operatorname{Ker} a$, so $u_{\left(\tau^{*}, \tau\right)}=1$.

Similarly, if for all $\left(\sigma^{*}, \sigma\right)$ :

$$
u_{\left(\tau^{*}, \tau\right)} u_{\left(\sigma^{*}, \sigma\right)}=p_{1} a\left(\tau^{*}, \tau\right) u_{\left(\sigma^{*}, \sigma\right)} u_{\left(\tau^{*}, \tau\right)},
$$

then the right-hand side is equal to

$$
\left\langle a\left(\tau^{*}, \tau\right), \Phi a\left(\sigma^{*}, \sigma\right)\right\rangle\left\langle\left(\sigma^{*}, \sigma\right), d\left(\tau^{*}, \tau\right)\right\rangle u_{\left(\tau^{*}, \tau\right)} u_{\left(\sigma^{*}, \sigma\right)},
$$

and

$$
0=\left(a^{*} \Phi^{*} a+d\right)\left(\tau^{*}, \tau\right)=a\left(\tau^{*}, \tau\right)
$$

so $u_{\left(\tau^{*}, \tau\right)}=1$.

Now $A$ is also $R$-separable, because $\left.\mid\left(G^{*} \times G\right) / \operatorname{Ker} a\right) \mid$ is invertible in $R$. So $A$ is a $R$ - $G$-Azumaya algebra, by 1.9 .

(3.4.7) One thing is left to be shown: $\beta([A])=f$, or $\beta_{A}\left(\sigma, \sigma^{*}\right)=f\left(\sigma, \sigma^{*}\right)$, or $\left(I-\alpha_{A}\right)\left(\sigma, \sigma^{*}\right)=(I-a t)\left(\sigma, \sigma^{*}\right)$, or $\alpha_{A}\left(\sigma, \sigma^{*}\right)=a\left(\sigma^{*}, \sigma\right)$. Let us show that

$$
\Phi_{A}\left(\sigma, \sigma^{*}\right)=\left\{x \in A: \beta(x) b=\left(\sigma, \sigma^{*}\right)(b) x,\right.
$$

for all homogeneous $b$ of degree $\beta$ \} 
is generated by $u_{\left(\sigma^{*}, \sigma\right)}$. Indeed, take $\left(\tau^{*}, \tau\right) \in G^{*} \times G$. Then $\operatorname{deg}_{G} u_{\left(\tau^{*}, \tau\right)}=$ $p_{1} a\left(\tau^{*}, \tau\right)$, and

and

$$
\left(p_{1} a\left(\tau^{*}, \tau\right)\right)\left(u_{\left(\sigma^{*}, \sigma\right)}\right) u_{\left(\tau^{*}, \tau\right)}=\left\langle a\left(\tau^{*}, \tau\right), \Phi a\left(\sigma^{*}, \sigma\right)\right\rangle u_{\left(\sigma^{*}, \sigma\right)} u_{\left(\tau^{*}, \tau\right)},
$$

$$
\begin{aligned}
\left(\sigma, \sigma^{*}\right)\left(u_{\left(\tau^{*}, \tau\right)}\right) u_{\left(\sigma^{*}, \sigma\right)} & =\left\langle\left(\sigma, \sigma^{*}\right), \operatorname{ta}\left(\tau^{*}, \tau\right)\right\rangle\left\langle\left(\tau^{*}, \tau\right), d\left(\sigma^{*}, \sigma\right)\right\rangle u_{\left(\sigma^{*}, \sigma\right)} u_{\left(\tau^{*}, \tau\right)} \\
& =\left\langle\left(a^{*}+d\right)\left(\sigma^{*}, \sigma\right),\left(\tau^{*}, \tau\right)\right\rangle u_{\left(\sigma^{*}, \sigma\right)} u_{\left(\tau^{*}, \tau\right)} \\
& =\left\langle\left(a^{*} \Phi a\right)\left(\sigma^{*}, \sigma\right),\left(\tau^{*}, \tau\right)\right\rangle u_{\left(\sigma^{*}, \sigma\right)} u_{\left(\tau^{*}, \tau\right)}
\end{aligned}
$$

Now $\operatorname{deg}_{G \times G^{*}} u_{\left(\sigma^{*}, \sigma\right)}=a\left(\sigma^{*}, \sigma\right)$, so $\alpha_{A}\left(\sigma, \sigma^{*}\right)=a\left(\sigma^{*}, \sigma\right)$, and this shows that $f \in \operatorname{Im} \beta$.

(3.4.8) Finally take $f \in O(R, G)_{\min }$. Then $f$ is locally constant on $\operatorname{Spec}(R)$, so there exist idempotents $e_{1}, \ldots, e_{n}$ such that $f$ is constant on $\operatorname{Spec}\left(\operatorname{Re}_{i}\right)$. Applying the above arguments to $\operatorname{BD}\left(R e_{i}, G\right)$, the result follows.

In the sequel, the algebra $A$ defined above will be denoted by $A_{f}$. As a first application let us determine the center of $A_{f}$. It is clear that $u_{\left(\sigma^{*}, \sigma\right)} \in Z\left(A_{f}\right)$ if $\left(\sigma^{*}, \sigma\right) \in \operatorname{Ker} d$. Ker $d$ is described by the following:

3.5. Lemma. Under the assumptions of Theorem 3.4, we have

$\operatorname{Ker} d=\operatorname{Ker}(I-\Phi a) \oplus \operatorname{Ker} a$.

Proof. It is clear that $\operatorname{Ker} a \cap \operatorname{Ker}(I-\Phi a)=0$. Also

$$
\operatorname{Ker} a=\operatorname{Ker} a^{*} \subset \operatorname{Ker}\left(a^{*} \Phi a-a^{*}\right)=\operatorname{Ker} d
$$

and

$$
\operatorname{Ker}(I-\Phi a) \subset \operatorname{Ker} a^{*}(I-\Phi a)=\operatorname{Ker} d .
$$

Take $\left(\sigma^{*}, \sigma\right) \in \operatorname{Ker} d$. Then $a^{*}(\Phi a-I)\left(\sigma^{*}, \sigma\right)=0$, so $(\Phi a-I)\left(\sigma^{*}, \sigma\right) \in$ $\operatorname{Ker} a^{*}=\operatorname{Ker} a$, and $a(\Phi a-I)\left(\sigma^{*}, \sigma\right)=0$, so $\Phi a(\Phi a-I)\left(\sigma^{*}, \sigma\right)=0$ and $\Phi a\left(\sigma^{*}, \sigma\right) \in \operatorname{Ker}(I-\Phi a)$. Finally

$$
\left(\sigma^{*}, \sigma\right)=\Phi a\left(\sigma^{*}, \sigma\right)+(I-\Phi a)\left(\sigma^{*}, \sigma\right) \in \operatorname{Ker}(I-\Phi a)+\operatorname{Ker} a .
$$

\subsection{Corollary. Under the assumptions of Theorem 3.4:}

(3.6.1) $Z\left(A_{f}\right)=R \operatorname{Ker}(I-\Phi a)$;

(3.6.2) A G-Azumaya algebra is Azumaya if and only if $I-\Phi a=I-$ $\Phi\left(I-\beta_{A}\right) t^{-1}$ is invertible.

Let us now discuss the relation with Orzech's map. Under the assumption that $G$ has exponent $m, \operatorname{Pic}(R)=1, R$ contains a primitive $m$ th root of unity and $H^{2}\left(G, \mathbf{G}_{\mathbf{m}}(R)\right)=1$, Orzech defined a map $\beta_{\text {Orz }}: \operatorname{BAz}(R, G) \rightarrow \operatorname{Aut}(G)$ (cf. $[28,4,14,7])$. $\quad \beta_{\text {Orz }}$ is surjective, and a preimage of $j \in \operatorname{Aut}(G)$ may be constructed as follows. Consider the groupring $R G$, furnished with its natural grading, and with $G$-action defined by $\sigma\left(u_{\tau}\right)=u_{\tau+k(\sigma)}$, where $k=I-j$. The $G$-graded $G$-module thus obtained is denoted by $R G(j)$. It may be shown that End $R G(j)$, with the induced action and grading is a $G$-Azumaya algebra (cf. loc. cit.). The following proposition relates $\beta_{\text {Orz }}$ to our map $\beta$ : 
3.7. Proposition. Let $R$ be a connected ring. Under the assumptions of 3.2, and with the above notations, we have that

$$
\beta([\text { End } R G(j)])=\left(j, j^{*-1}\right) \in \operatorname{Aut}\left(G \times G^{*}\right) .
$$

Proof. First, take $\sigma \in G$, and let $x_{\sigma}$ be the automorphism of $A=\operatorname{End} R G(j)$ given by $x_{\sigma}(f)=\sigma(f)$. Then $\operatorname{deg} x_{\sigma}=k(\sigma), G$ acts trivially on $x_{\sigma}$, and $x_{\sigma}$ induces the action of $\sigma$ on $A$. So $\alpha_{A}(\sigma, 0)=(k(\sigma), 0)$ and $\beta_{A}(\sigma, 0)=$ $(j(\sigma), 0)$. Next, take $\sigma^{*} \in G^{*}$. Now, let $x_{\sigma^{*}}$ be the automorphism of $R G(j)$ given by $x_{\sigma^{*}}\left(u_{\tau}\right)=\sigma^{*}\left(j^{-1}(\tau)\right) u_{\tau}$. It is clear that $\operatorname{deg} x_{\sigma^{*}}=0$. We describe the action of $G$ on $x_{\sigma^{*}}$. For all $\alpha, \tau \in G$, we have

$$
\begin{aligned}
\alpha\left(x_{\sigma^{*}}\right) u_{\tau} & =\alpha\left(x_{\sigma^{*}}\left(\alpha^{-1}\left(u_{\tau}\right)\right)\right)=\alpha\left(x_{\sigma^{*}}\left(u_{\tau-k(\alpha)}\right)\right) \\
& =\sigma^{*}\left(j^{-1}(\tau-k(\alpha)) \alpha\left(u_{\tau-k(\alpha)}\right)\right. \\
& =\sigma^{*}\left(j^{-1}(\tau-k(\alpha)) \sigma^{*}\left(j^{-1}(\tau)\right)^{-1} x_{\sigma^{*}}\left(u_{\tau}\right)\right. \\
& =\sigma^{*}\left(j^{-1}(-k(\alpha)) x_{\sigma^{*}}\left(u_{\tau}\right)\right. \\
& =\sigma^{*}\left(\alpha-j^{-1}(\alpha)\right) x_{\sigma^{*}}\left(u_{\tau}\right),
\end{aligned}
$$

so $\alpha\left(x_{\sigma^{*}}\right)=\sigma^{*}\left(\alpha-j^{-1}(\alpha)\right) x_{\sigma^{*}}=\left(I-j^{-1}\right)^{*}\left(\sigma^{*}\right)(\alpha) x_{\sigma^{*}}$. Let us show that $x_{\sigma^{*}} \in \Phi_{A}\left(0, \sigma^{*}\right)$. Let $a \in A$ be homogeneous of degree $\alpha$, and take $\tau \in G$. Then

$$
\begin{aligned}
\alpha\left(x_{\sigma^{*}}\right)(a)\left(u_{\tau}\right) & =\sigma^{*}\left(\alpha-j^{-1}(\alpha)\right) x_{\sigma^{*}}(a)\left(u_{\tau}\right) \\
& =\sigma^{*}\left(\alpha-j^{-1}(\alpha)\right) \sigma^{*}\left(j^{-1}(\alpha+\tau)\right) a\left(u_{\tau}\right) \\
& =\sigma^{*}\left(\alpha+j^{-1}(\tau)\right) a\left(u_{\tau}\right),
\end{aligned}
$$

and

$$
\sigma^{*}(a) x_{\sigma^{*}}\left(u_{\tau}\right)=\sigma^{*}(\alpha) \sigma^{*}\left(j^{-1}(\tau)\right) a u_{\tau} .
$$

It therefore follows that

$$
\alpha_{A}\left(0, \sigma^{*}\right)=\left(0,\left(\left(I-j^{-1}\right)^{*}\right)\left(\sigma^{*}\right)\right), \quad \beta_{A}\left(0, \sigma^{*}\right)=\left(0,\left(j^{-1}\right)^{*}(\sigma)\right) .
$$

3.8. Example. Let $R$ be a connected ring, and assume that 2 is invertible in $R$. Let $G=\mathbf{Z} / 2 \mathbf{Z}=\langle\sigma\rangle$. The group ring $A=R G$ with action defined by $\sigma\left(u_{\sigma}\right)=-u_{\sigma}$ is a $G$-Azumaya algebra. $G \times G^{*}=\mathbf{Z} / 2 \mathbf{Z} \times \mathbf{Z} / 2 \mathbf{Z}$, and the automorphism $\beta_{A}$ is given by its matrix representation:

$$
\beta_{A}=\left(\begin{array}{ll}
0 & 1 \\
1 & 0
\end{array}\right) .
$$

We leave the verification of this to the reader.

3.9. Example. Let $R$ be a connected ring, and assume that 2 is invertible in $R$. Let $G=\mathbf{Z} / 2 \mathbf{Z} \times \mathbf{Z} / 2 \mathbf{Z}=\langle\sigma\rangle \times\langle\tau\rangle$. In [28], Orzech gave an example of a $G$-Azumaya algebra $A$ which is central, and such that its $G$-opposite algebra $\bar{A}$ is commutative. Indeed, from 3.6, it follows that there is no reason why $A$ 
central should imply $\bar{A}$ central. Let us work out Orzech's example to illustrate this. Let $A$ be equal to $R G$ as a graded $R$-module, and let the multiplication rule be given by

Define the $G$-action by

$$
u_{\sigma} u_{\tau}=-u_{\tau} u_{\sigma}=u_{\sigma+\tau}
$$

$$
\sigma\left(u_{\sigma}\right)=\tau\left(u_{\sigma}\right)=-u_{\sigma} \text { and } \sigma\left(u_{\tau}\right)=-\tau\left(u_{\tau}\right)=u_{\tau} .
$$

Let $\sigma^{*}, \tau^{*} \in G^{*}$ be defined by $\sigma^{*}(\sigma)=\tau^{*}(\tau)=-1, \sigma^{*}(\tau)=\tau^{*}(\sigma)=1 . G \times G^{*}$ is of rank 4 over $\mathbf{Z} / 2 \mathbf{Z}$, and will be identified with $\langle\sigma\rangle \times\langle\tau\rangle \times\left\langle\sigma^{*}\right\rangle \times\left\langle\tau^{*}\right\rangle$. The action of $G^{*}$ is given by

$$
\sigma^{*}\left(u_{\sigma}\right)=-\tau^{*}\left(u_{\sigma}\right)=-u_{\sigma} \text { and } \sigma^{*}\left(u_{\tau}\right)=-\tau^{*}\left(u_{\tau}\right)=u_{\tau} .
$$

A direct verification shows that the action of $\sigma$ and $\sigma^{*}$ are induced by $u_{\sigma}$, the action of $\tau$ by $u_{\tau}$, and the action of $\tau^{*}$ by $u_{\sigma+\tau}$. Therefore $\alpha_{A}$ and $\beta_{A}$ are given by the matrices

$$
\alpha_{A}=\left(\begin{array}{cccc}
1 & 0 & 1 & 1 \\
0 & 1 & 0 & 1 \\
1 & 0 & 1 & 1 \\
1 & 1 & 1 & 0
\end{array}\right) ; \quad \beta_{A}=\left(\begin{array}{cccc}
0 & 0 & 1 & 1 \\
0 & 0 & 0 & 1 \\
1 & 0 & 0 & 1 \\
1 & 1 & 1 & 1
\end{array}\right) .
$$

We can now compute that

$$
I-\Phi \alpha_{A} t^{-1}=\left(\begin{array}{cccc}
0 & 1 & 1 & 0 \\
1 & 1 & 1 & 0 \\
0 & 0 & 1 & 0 \\
0 & 0 & 0 & 1
\end{array}\right)
$$

is invertible, as expected. Also

$$
\beta_{A}^{-1}=t^{-1} \beta_{A}^{*} t=\left(\begin{array}{llll}
0 & 1 & 1 & 0 \\
1 & 1 & 1 & 1 \\
1 & 1 & 0 & 0 \\
0 & 1 & 0 & 0
\end{array}\right)
$$

and

$$
I-\Phi\left(I-\beta_{A}^{-1}\right) t^{-1}=\left(\begin{array}{cccc}
0 & 0 & 1 & 1 \\
0 & 0 & 0 & 1 \\
0 & 0 & 1 & 0 \\
0 & 0 & 0 & 1
\end{array}\right)
$$

is of rank 2; so the center of $\bar{A}$ is of rank 2 over $R$, as expected.

3.10. Example. From the results of Long [23, Theorem 2.7], it follows that $O(R, \mathbf{Z} / p \mathbf{Z})_{\min }$ is the dihedral group of $2(p-1)$ elements (if $R$ is connected, and if $R$ contains $p^{-1}$ and a primitive $p$ th root of unity). We leave it as an exercise to the reader to deduce this from the above results.

\section{GALOIS EXTENSIONS AND STRONGLY GRADED RINGS}

In this section, we discuss the following subgroups of $\operatorname{BD}(R, G): \operatorname{BM}(R, G)$ and $\mathrm{BC}(R, G)$, the subgroups of Brauer-Long classes represented respectively 
by algebras with trivial grading and trivial action. Their importance lies in their connection to Galois theory. It has been shown by the second author [3, Theorem 1.4] that

$$
\begin{aligned}
\mathrm{BM}(R, G) & \cong \mathrm{Br}(R) \times \mathrm{Gal}(R, R G), \\
\mathrm{BC}(R, G) & \cong \mathrm{Br}(R) \times \mathrm{Gal}(R, G R) .
\end{aligned}
$$

4.1. Theorem. We have exact sequences

$$
\begin{aligned}
1 & \rightarrow \mathrm{BM}^{s}(R, G) \rightarrow \mathrm{BM}(R, G) \\
& \stackrel{\beta}{\rightarrow}\left\{f \in \operatorname{Hom}\left(G, G^{*}\right)(R):\langle\sigma, f(\sigma)\rangle=1, \text { for all } \sigma \in G(R)\right\}, \\
1 & \rightarrow \mathrm{BC}^{s}(R, G) \rightarrow \mathrm{BC}(R, G) \\
& \stackrel{\beta}{\rightarrow}\left\{f \in \operatorname{Hom}\left(G^{*}, G\right)(R):\left\langle\sigma^{*}, f\left(\sigma^{*}\right)\right\rangle=1, \text { for all } \sigma^{*} \in G^{*}(R)\right\} .
\end{aligned}
$$

The maps $\beta$ are surjective if $G(R) \cong G^{* *}(R)$ and if $|G|$ is invertible. The isomorphism of Theorem 2.7 restricts to

$$
\mathrm{BM}^{S}(R, G) \cong H^{1}\left(R_{\mathrm{fl}}, G^{*}\right) \times \mathrm{Br}(R) \text { and } \mathrm{BC}^{s}(R, G) \cong H^{1}\left(R_{\mathrm{et}}, G\right) \times \mathrm{Br}(R) \text {. }
$$

Proof. Take $[A] \in \operatorname{BM}(R, G)$, and consider the map $\beta$ defines in $\S 3$. Since $A$ has trivial grading, we have that $\alpha_{A}\left(\sigma, \sigma^{*}\right)=(0, f(\sigma))$, for some $f \in$ $\operatorname{Hom}\left(G, G^{*}\right)(R)$ and therefore $\beta_{A}$ is given by the matrix

$$
\beta_{A}=\left(\begin{array}{ll}
I & 0 \\
f & I
\end{array}\right) \text {. }
$$

It is easily verified that a matrix of type (4.1.3) defines an automorphism in $O(R, G)_{\min }$ if and only if $\langle\sigma, f(\sigma)\rangle=1$, for all $\sigma \in G(R)$, and the first exact sequence follows. The second one is obtained in a similar way. The ontoness of $\beta$ follows from Theorem 3.4. The second statement follows immediately from Theorem 2.7 or from 1.13 and [3].

4.2. Proposition. Suppose that $G(R) \cong G^{* *}(R)$ and that $|G|^{-1} \in R$. Then we have split short exact sequences

$$
\begin{aligned}
1 & \rightarrow \operatorname{Gal}^{s}(R, R G) \rightarrow \operatorname{Gal}(R, R G) \\
& \rightarrow\left\{f \in \operatorname{Hom}\left(G, G^{*}\right)(R):\langle\sigma, f(\sigma)\rangle=1, \text { for all } \sigma \in G(R)\right\} \rightarrow 1,
\end{aligned}
$$

$$
\begin{aligned}
1 & \rightarrow \mathrm{Gal}^{s}(R, G R) \rightarrow \operatorname{Gal}(R, G R) \\
& \rightarrow\left\{f \in \operatorname{Hom}\left(G^{*}, G\right)(R):\left\langle\sigma^{*}, f\left(\sigma^{*}\right)\right\rangle=1, \text { for all } \sigma^{*} \in G^{*}(R)\right\} \rightarrow 1 .
\end{aligned}
$$

Proof. The exactness of the sequences follows from (4.1.1-4.1.2), 1.13 and 4.1. We have the following commutative diagram:

$$
\begin{aligned}
& 1 \rightarrow \mathrm{BM}^{s}(R, G) \rightarrow \operatorname{BM}(R, G) \stackrel{\beta}{\rightarrow}\left\{f \in \operatorname{Hom}\left(G, G^{*}\right)(R):\langle\sigma, f(\sigma)\rangle=1 \text { for all } \sigma \in G(R)\right\} \rightarrow 1 \\
& \downarrow \pi \quad \downarrow \pi \quad \downarrow \cong \\
& 1 \rightarrow \mathrm{Gal}^{s}(R, R G) \rightarrow \operatorname{Gal}(R, R G) \stackrel{\alpha}{\rightarrow}\left\{f \in \operatorname{Hom}\left(G, G^{*}\right)(R):\langle\sigma: f(\sigma)\rangle=1 \text { for all } \sigma \in G(R)\right\} \rightarrow 1 \text {. }
\end{aligned}
$$


The map $\pi$ is defined as follows: for $[A] \in \operatorname{BM}(R, G), \pi([A])=(A \# R G)^{A}=$ $\{x \in A \# R G:(a \# 1) x=x(a \# 1)$, for all $a \in A\}$, cf. [3, $\S 2.1] . \alpha$ is then defined by commutativity of the above diagram. Let us consider the case where $R$ is connected. Take $f \in \operatorname{Hom}\left(G, G^{*}\right)$ such that $\langle\sigma, f(\sigma)\rangle=1$ for all $\sigma \in G$. We claim that a preimage $S_{f}$ of $f$ under $\alpha$ is given by the following data: $S_{f}=R G$ as a strongly graded $R$-module, and the multiplication on $S_{f}$ is defined by

$$
w_{\sigma} w_{\tau}=\langle\sigma, f(\tau)\rangle w_{\tau} w_{\sigma} ; \quad w_{\sigma}^{n}=w_{n \sigma} .
$$

Let us show that $\alpha\left(\left[S_{f}\right]\right)=f$. Let $g \in \operatorname{Aut}\left(G \times G^{*}\right)$ be defined by the matrix

$$
g=\left(\begin{array}{ll}
I & 0 \\
f & I
\end{array}\right) \text {. }
$$

Consider the preimage $A_{g}$ of $g$ in $\operatorname{BM}(R, G)$ as constructed in (3.4.5). We will show that $\pi\left(\left[A_{g}\right]\right)=S_{f}$. As in (3.4.5), let $a=(I-g) t^{-1}, d=a^{*} \Phi a-a^{*}$. Then we can verify easily that

$$
d=\left(\begin{array}{cc}
0 & 0 \\
0 & f^{*}
\end{array}\right) ; \quad a=\left(\begin{array}{cc}
0 & 0 \\
0 & -f
\end{array}\right) .
$$

As an $R$-module $A_{g}=R\left(\left(G^{*} \times G\right) / \operatorname{Ker} a\right) \cong R G / \operatorname{Ker} f$. Following (3.4.5), action and grading on $A_{g}$ are given by $\operatorname{deg}_{G} u_{\sigma}=p_{1} a(0, \sigma)=0$, as expected, and $\tau\left(u_{\sigma}\right)=\langle(\tau, 0), \Phi a(0, \sigma)\rangle u_{\sigma}=\left\langle(\tau, 0),(0,-f(\sigma)\rangle u_{\sigma}=\langle\tau,-f(\sigma)\rangle u_{\sigma}\right.$, while the multiplication is given by $u_{\sigma} u_{\tau}=\langle f(\sigma), \tau\rangle u_{\tau} u_{\sigma}$. To compute $\pi\left(\left[A_{g}\right]\right)$, let $\left\{v_{\sigma}: \sigma \in G\right\}$ be a basis for $R G$. In $A \# R G$, we have that

$$
\left(u_{\sigma} \# v_{\mu}\right)\left(u_{\tau} \# v_{\nu}\right)=\langle\tau,-f(\mu)\rangle u_{\sigma} u_{\tau} \# v_{\mu} v_{\nu} .
$$

It follows that

$$
\begin{gathered}
\left(u_{\sigma} \# 1\right)\left(u_{\tau} \# v_{\nu}\right)=u_{\sigma} u_{\tau} \# v_{\nu}, \\
\left(u_{\tau} \# v_{\nu}\right)\left(u_{\sigma} \# 1\right)=\langle\sigma,-f(\nu)\rangle u_{\tau} u_{\sigma} \# v_{\nu}=\langle\sigma, f(\tau)-f(\nu)\rangle u_{\sigma} u_{\tau} \# v_{\nu} .
\end{gathered}
$$

Hence $u_{\tau} \# v_{v} \in \pi\left(\left[A_{g}\right]\right)$ if and only if $f(\tau)=f(v)$. Since $u_{\sigma}=u_{\sigma+\xi}$ if $\xi \in \operatorname{Ker} f, \pi\left(\left[A_{g}\right]\right)$ is generated over $R$ by $\left\{w_{\sigma}=u_{\sigma} \# v_{\sigma}: \sigma \in G\right\}$. Now we leave it to the reader as a straightforward exercise to show that

$$
w_{\sigma} w_{\tau}=\langle\sigma, f(\tau)\rangle w_{\tau} w_{\sigma} .
$$

Hence $\pi\left(\left[A_{g}\right]\right)=S_{f}$, and $\alpha\left(S_{f}\right)=f$. Also it is clear that $\left[S_{f}\right]\left[S_{f^{\prime}}\right]=\left[S_{f+f^{\prime}}\right]$ in $\operatorname{Gal}(R, R G)$ so the proof of the splitting of the first exact sequence is complete. (4.2.2) is handled in a similar way.

4.3. Proposition (Generalization of the Kummer exact sequence). Let $R$ be a commutative ring, and $G$ a finite abelian group, then we have an exact sequence

$$
1 \rightarrow H_{\text {symm }}^{2}\left(G, \mathbf{G}_{\mathbf{m}}(R)\right) \rightarrow \mathrm{Gal}^{s}(R, R G) \rightarrow H^{1}(G, \operatorname{Pic}(R)) .
$$

If $G(R) \cong G^{* *}(R)$ and $|G|^{-1} \in R$, then we also have

$$
1 \rightarrow H_{\text {symm }}^{2}\left(G^{*}, \mathbf{G}_{\mathbf{m}}(R)\right) \rightarrow \operatorname{Gal}^{s}(R, G R) \rightarrow H^{1}\left(G^{*}, \operatorname{Pic}(R)\right) .
$$


Proof. Take a faithfully flat $R$-algebra $S$, and consider the exact sequence

$$
1 \rightarrow \operatorname{Hom}\left(G, \mathbf{G}_{\mathbf{m}}(S)\right) \rightarrow K^{1}\left(G, \mathbf{G}_{\mathbf{m}}(S)\right) \rightarrow Z_{\text {symm }}^{2}\left(G, \mathbf{G}_{\mathbf{m}}(S)\right) .
$$

Every symmetric cocycle can be split by a faithfully flat extension; therefore, we have an exact sequence of sheaves on $R_{\mathrm{fl}}$ :

$$
1 \rightarrow G^{*}(\cdot) \rightarrow K^{1}\left(G, \mathbf{G}_{\mathbf{m}}(\cdot)\right) \rightarrow Z_{\text {symm }}^{2}\left(G, \mathbf{G}_{\mathbf{m}}(\cdot)\right) \rightarrow 1 .
$$

We therefore have a long exact sequence of groups:

$$
\begin{aligned}
1 & \rightarrow G^{*}(R) \rightarrow K^{1}\left(G, \mathbf{G}_{\mathbf{m}}(R)\right) \rightarrow Z_{\text {symm }}^{2}\left(G, \mathbf{G}_{\mathbf{m}}(R)\right) \\
& \rightarrow H^{1}\left(R_{\mathrm{fl}}, G^{*}\right) \rightarrow K^{1}(G, \operatorname{Pic}(R)) \rightarrow \cdots .
\end{aligned}
$$

It follows that we have a short exact sequence:

$$
1 \rightarrow H_{\text {symm }}^{2}\left(G, \mathbf{G}_{\mathbf{m}}(R)\right) \rightarrow H^{1}\left(R_{\mathrm{fl}}, G^{*}\right) \cong \operatorname{Gal}^{s}(R, R G) \rightarrow H^{1}(G, \operatorname{Pic}(R)) .
$$

The second sequence is obtained in a similar way.

If $G=\mathbf{Z} / n \mathbf{Z}, n$ is invertible in $R$ and $R$ contains a primitive $n$th root of unity, then $G \cong G^{* *}$ and the sequence above reduces to the well-known Kummer sequence

$$
1 \rightarrow \mathbf{G}_{\mathbf{m}}(R) /\left(\mathbf{G}_{\mathbf{m}}(R)\right)^{n} \rightarrow T(R, G) \rightarrow_{n} \operatorname{Pic}(R) \rightarrow 1 .
$$

4.4. Remark. In the notations of Proposition 4.3, $f$ defines a 2-cocycle $h \in$ $Z^{2}\left(G^{*}, \mathbf{G}_{\mathbf{m}}(R)\right)$, by the rule

$$
h\left(\sigma^{*}, \tau^{*}\right)=u_{\sigma^{*}+\tau^{*}} u_{\sigma^{*}}^{-1} u_{\tau^{*}}^{-1} .
$$

The inverse image of $f$ defined above is nothing else than the crossed product $\left(R, G^{*}, h\right)$. The cocycle $h$ is a skew pairing in the terminology of DeMeyer [15]. If $\operatorname{Pic}(R)=1$, then (4.2.2) is isomorphic to DeMeyer's split exact sequence [15, Proposition 1] (use 4.3):

$$
1 \rightarrow H_{\text {symm }}^{2}\left(G, \mathbf{G}_{\mathbf{m}}(R)\right) \rightarrow H^{2}\left(G, \mathbf{G}_{\mathbf{m}}(R)\right) \rightarrow P_{\text {sk }}\left(G, \mathbf{G}_{\mathbf{m}}(R)\right) \rightarrow 1 .
$$

\section{The graded Brauer group of Childs, Garfinkel and Orzech}

In [13], Childs, Garfinkel and Orzech defined a generalized Brauer group of classes of algebras graded by a finite abelian group $G$ and with $G$-action defined via a bilinear map $\varphi: G(R) \times G(R) \rightarrow \mathbf{G}_{\mathbf{m}}(R)$. This group $B_{\varphi}(R, G)$ is a subgroup of $\operatorname{BD}(R, G)$ consisting of those classes $[A]$ in $\operatorname{BD}(R, G)$ for which the $G$-action on $A$ is determined by the $G$-grading as follows: $\sigma(a)=$ $\varphi(\sigma, \operatorname{deg} a) a$, for all homogeneous $a \in A$. If $\varphi$ is the trivial bilinear map, then $B_{\varphi}(R, G)=\mathrm{BC}(R, G)$, and if $G=\mathbf{Z} / 2 \mathbf{Z}$, and if $\varphi$ is the nontrivial bilinear map, then $B_{\varphi}(R, G)=\mathrm{BW}(R)$, the Brauer-Wall group. The results of the previous sections may be applied to describe $B_{\varphi}(R, G)$. As one might expect, we start off with a description of $B_{\varphi}^{S}(R, G)=B_{\varphi}(R, G) \cap \mathrm{BD}^{S}(R, G)$. 
5.1. Proposition. Let $R$ be a commutative ring, $G$ a finite abelian group, and $\varphi: G(R) \times G(R) \rightarrow \mathbf{G}_{\mathbf{m}}(R)$ a bilinear map. Then we have that

$$
B_{\varphi}^{s}(R, G) \cong H^{1}\left(R_{\mathrm{et}}, G\right) \times H^{2}\left(R_{\mathrm{et}}, \mathbf{G}_{\mathrm{m}}\right)_{\mathrm{tors}},
$$

where the multiplication rules are given by

$$
(\sigma, u)(\tau, v)=(\sigma+\tau, \Phi(\sigma, \tau) u v) .
$$

$\Phi$ is defined by the maps $G\left(S^{(2)}\right) \times G\left(S^{(2)}\right) \rightarrow \mathbf{G}_{\mathbf{m}}\left(S^{(3)}\right):(\sigma, \tau) \rightarrow \varphi\left(\sigma_{1}, \tau_{3}\right)$.

Proof. Observe that $\varphi$ induces a bilinear map of sheaves $\varphi: G(\cdot) \times G(\cdot) \rightarrow$ $\mathbf{G}_{\mathbf{m}}(\cdot)$, and defines a homomorphism of sheaves $\Psi: G(R) \rightarrow G^{*}(R): \sigma \rightarrow(\tau \rightarrow$ $\varphi(\sigma, \tau))$. Inspecting the proof of Theorem 2.7, we obtain (this becomes very clear if we look at the remark made in 2.8):

$$
\begin{aligned}
B_{\varphi}^{s}(R, G) & \cong\left\{\left(\sigma, \sigma^{*}, \mathcal{u}\right): \sigma^{*}=\psi(\sigma)\right\} \\
& \cong H^{1}\left(R_{\text {ét }}, G\right) \times H^{2}\left(R_{\text {êt }}, \mathbf{G}_{\mathbf{m}}\right)_{\text {tors }} .
\end{aligned}
$$

Also, if $\sigma$ and $\tau$ are represented by some $\sigma$ and $\tau \in Z^{1}(S / R, G)$ for some étale covering $S$ of $R$ :

$$
\begin{aligned}
(\sigma, \psi(\sigma), u)(\tau, \psi(\tau), v) & =\left(\sigma+\tau, \psi(\sigma+\tau), \psi\left(\sigma_{1}\right)\left(\tau_{3}\right) u v\right) \\
& =\left(\sigma+\tau, \psi(\sigma+\tau), \varphi\left(\sigma_{1}, \tau_{3}\right) u v\right) .
\end{aligned}
$$

Our next aim is apply 3.4 to describe $B_{\varphi}(R, G)$ by a short exact sequence. We modify the map $\beta$ of 3.4 to obtain an epimorphism $\beta_{\varphi}$ from $B_{\varphi}(R, G)$ onto a subgroup of $\operatorname{Hom}\left(G^{*}, G\right)(R)$. We define $O_{\varphi}(R, G)_{\min }=\{f \in$ $\operatorname{Hom}\left(G^{*}, G\right)(R):\left\langle\sigma^{*},\left(f-f^{*} \psi f\right)\left(\sigma^{*}\right)\right\rangle=1$ for all $\left.\sigma^{*} \in G(R)\right\}$; the multiplication on $O_{\varphi}(R, G)_{\min }$ is defined by $f \cdot f^{\prime}=f+f^{\prime}-f\left(\psi+\psi^{*}\right) f^{\prime}$.

5.2. Theorem. Assume that $G(R) \cong G^{* *}(R)$ and that $|G|$ is invertible in $R$. Then $B_{\varphi}(R, G)$ is described by the following exact sequence:

$$
1 \rightarrow B_{\varphi}^{s}(R, G) \rightarrow B_{\varphi}(R, G) \stackrel{\beta_{\varphi}}{\longrightarrow} O_{\varphi}(R, G)_{\min } \rightarrow 1 .
$$

Proof. Let $A$ represent an element of $B_{\varphi}(R, G)$. Then $\alpha_{A}$ and $\beta_{A}$ can be written as matrices:

$$
\begin{gathered}
\alpha_{A}=\left(\begin{array}{ll}
a_{11} & a_{12} \\
a_{21} & a_{22}
\end{array}\right), \\
\beta_{A}=I-\alpha_{A}=\left(\begin{array}{ll}
b_{11} & b_{12} \\
b_{21} & b_{22}
\end{array}\right) \in \operatorname{Hom}\left(\begin{array}{cc}
(G, G) & \left(G^{*}, G\right) \\
\left(G, G^{*}\right) & \left(G^{*}, G^{*}\right)
\end{array}\right) .
\end{gathered}
$$

Now, for all $\sigma \in G(R), \sigma(a)=\psi(\sigma)(a)$, and therefore

$$
\alpha_{A}(\sigma, 0)=\alpha_{A}(0, \psi(\sigma)) \text {. }
$$

It follows that $a_{11}=a_{12} \circ \psi, a_{21}=a_{22} \circ \psi$. Take $\sigma^{*} \in G^{*}$, and let $\alpha_{A}\left(0, \sigma^{*}\right)=$ $\left(\tau, \tau^{*}\right)$. Consider $I_{\left(0, \sigma^{*}\right)}$ as defined in Theorem 3.1. For $v \in I_{\left(0, \sigma^{*}\right)}$, and for all homogeneous $a$ in $A$, we have that $\sigma^{*}(a) v=\alpha(v) a$, where $\operatorname{deg} a=\alpha$. 
We have that $\alpha(v)=\psi(\alpha)(\tau) v=\tau^{*}(\alpha) v$. So $\langle\psi(\alpha), \tau\rangle=\left\langle\tau^{*}, \alpha\right\rangle$ for all $\alpha \in G$, so $\tau^{*}=\psi^{*}(\tau)$, or $a_{22}\left(\sigma^{*}\right)=\psi^{*}\left(a_{12}\left(\sigma^{*}\right)\right)$, so $a_{22}=\psi^{*}\left(a_{12}\right)$. We set $\beta_{\varphi}([A])=f=a_{12}=b_{12} \in \operatorname{Hom}\left(G^{*}, G\right)(R)$. Then it follows easily that

$$
\beta_{A}=\left(\begin{array}{cc}
I-f \psi & f \\
\psi^{*} f \psi & I-\psi^{*} f
\end{array}\right) .
$$

Applying Theorem 3.4, we obtain that it is necessary and sufficient for an automorphism of type (5.2.1) to lie in $\operatorname{Im} \beta$ that

$$
\begin{aligned}
\sigma^{*}(\sigma) & =\left\langle\beta_{A}\left(\sigma, \sigma^{*}\right), \Phi \beta_{A}\left(\sigma, \sigma^{*}\right)\right\rangle \\
& =\left\langle\psi^{*} f \psi(\sigma)+\sigma^{*}-\psi^{*} f\left(\sigma^{*}\right), \sigma-f \psi(\sigma)+f\left(\sigma^{*}\right)\right\rangle
\end{aligned}
$$

for all $\left(\sigma, \sigma^{*}\right)$. Setting $\sigma=0$ in (5.2.2), we obtain

$$
\left\langle\sigma^{*}-\psi^{*} f\left(\sigma^{*}\right), f\left(\sigma^{*}\right)\right\rangle=1,
$$

or

$$
\left\langle\sigma^{*},\left(f-f^{*} \psi f\right)\left(\sigma^{*}\right)\right\rangle=1 .
$$

Conversely, suppose that $(5.2 .3)$ holds for all $\sigma^{*} \in G^{*}(R)$. Then a little computation shows that $(5.2 .2)$ is equivalent to

$$
\begin{aligned}
\left\langle\sigma, \psi^{*} f \psi(\sigma)\right\rangle\left\langle\sigma, \psi^{*} f\left(\sigma^{*}\right)\right\rangle^{-1} & \left\langle f \psi(\sigma), \psi^{*} f \psi(\sigma)\right\rangle^{-1}\left\langle f \psi(\sigma), \sigma^{*}\right\rangle^{-1} \\
& \cdot\left\langle f \psi(\sigma), \psi^{*} f\left(\sigma^{*}\right)\right\rangle\left\langle f\left(\sigma^{*}\right), \psi^{*} f \psi(\sigma)\right\rangle=1 .
\end{aligned}
$$

The product of the first and the third factor equals (applying (5.2.3) with $\sigma^{*}=$ $\psi(\sigma))$

$$
\left\langle\psi(\sigma),\left(f-f^{*} \psi f\right) \psi(\sigma)\right\rangle=1 .
$$

Then (5.2.2) reduces to

$$
\left\langle\sigma, \psi^{*}\left(f+f^{*}-f^{*} \psi^{*} f-f^{*} \psi f\right)\left(\sigma^{*}\right)\right\rangle=1 .
$$

From (5.2.3), it follows that $f-f^{*} \psi f$ is skew, and then (5.2.2) follows. Finally, the multiplication rules are derived easily: take two matrices of the form (5.2.1), and multiply them, to find that the entry in the $(1,2)$-spot is given by $f \cdot f^{\prime}=$ $f+f^{\prime}-f\left(\psi+\psi^{*}\right) f^{\prime}$.

\subsection{Notes.}

(5.3.1) We have seen in the proof of Theorem 5.2 that $f \in O_{\varphi}(R, G)_{\min }$ satisfies the equation $f+f^{*}-f^{*}\left(\psi+\psi^{*}\right) f=0$. Therefore, the inverse of $f$ in $O_{\varphi}(R, G)_{\min }$ is given by $f^{*}$. If $|G|$ is not a multiple of 2 , then it is easily seen that $O_{\varphi}(R, G)_{\min }=O_{\varphi}(R, G)_{\max }=\left\{f \in \operatorname{Hom}\left(G^{*}, G\right)(R): f+f^{*}=\right.$ $\left.f^{*}\left(\psi+\psi^{*}\right) f\right\}$.

(5.3.2) Define $\gamma_{\varphi}: B_{\varphi}(R, G) \rightarrow \operatorname{Aut}\left(G^{*}\right)$ by $\gamma_{\varphi}([A])=I-f^{*} m$, where $f=$ $\beta_{\varphi}([A]), m=\psi+\psi^{*}$. It follows immediately from the multiplication rules in $O_{\varphi}(R, G)_{\min }$ that $\gamma_{\varphi}$ is a group homomorphism. Also $g=\gamma_{\varphi}([A])$ satisfies the 
equation $g^{*} m g=m$, and if $[A] \in \operatorname{Ker} \gamma_{\varphi}$, then $f^{*} m=0$. We therefore have the following exact sequences:

$$
\begin{gathered}
1 \rightarrow \operatorname{Ker} \gamma_{\varphi} \rightarrow \operatorname{BD}(R, G) \stackrel{\nu_{\varphi}}{\longrightarrow}\left\{g \in \operatorname{Aut}\left(G^{*}\right): g^{*} m g=m\right\} \\
1 \rightarrow \operatorname{BD}^{s}(R, G) \rightarrow \operatorname{Ker} \gamma_{\varphi} \stackrel{\beta_{\varphi}}{\longrightarrow}\left\{f \in \operatorname{Hom}\left(G^{*}, G\right): f+f^{*}=0, f^{*} m=0\right\} .
\end{gathered}
$$

If $m$ is invertible, then clearly $\beta_{\varphi}=0$, and we have the exact sequence:

$$
1 \rightarrow \mathrm{BD}^{s}(R, G) \rightarrow \mathrm{BD}(R, G) \stackrel{\gamma_{\varphi}}{\longrightarrow}\left\{g \in \operatorname{Aut}\left(G^{*}\right): g^{*} m g=m\right\}
$$

where $\gamma_{\varphi}([A])=I-\beta_{\varphi}([A])^{*} m$.

If $(|G|, 2)=1$, then $\gamma_{\varphi}$ is onto, and we obtain a generalization of another result of Childs $[12,3.4]$. If 2 divides the order of $G$, then we leave it to the reader to show that $\operatorname{Im} \gamma_{\varphi}=\left\{g \in \operatorname{Aut}\left(G^{*}\right):\left\langle\psi\left(\sigma^{*}\right), \sigma^{*}\right\rangle=\left\langle\psi g\left(\sigma^{*}\right), g\left(\sigma^{*}\right)\right\rangle\right.$, for all $\left.\sigma^{*} \in G^{*}\right\}$.

Acknowledgment. The first author would like to thank Mount Allison University for their hospitality during his visit of June, 1987, when the idea for this project was born. He would also like to thank F. DeMeyer and A. Bak for their helpful conversations and for their hospitality during his visits at Colorado State University and the University of Bielefeld.

\section{REFERENCES}

1. M. Artin, On the joins of Hensel rings, Adv. in Math. 7 (1971), 282-296.

2. H. Bass, Clifford algebras and spinor norms over a commutative ring, Amer. J. Math. 96 (1974), 156-206.

3. M. Beattie, A direct sum decomposition for the Brauer group of $H$-module algebras, $\mathbf{J}$. Algebra 43 (1976), 686-693.

4. _ The Brauer group of central separable G-Azumaya algebras, J. Algebra 54 (1978), 516-525.

5. __ Automorphisms of G-Azumaya algebras, Canad. J. Math. 37 (1985), 1047-1058. Correction to Automorphisms of G-Azumaya algebras (preprint).

6. __ Computing the Brauer group of graded Azumaya algebras from its subgroups, J. Algebra 101 (1986), 339-349.

7. M. Beattie and S. Caenepeel, The Brauer-Long group of $\mathbf{Z} / p^{t} \mathbf{Z}$-dimodule algebras, J. Pure Appl. Algebra 60 (1989), 219-236.

8. S. Caenepeel, A cohomological interpretation of the Brauer-Wall group, Proceeding of the Second Spanish-Belgian Week on Algebra and Geometry, Algebra, vol. 57, Santiago de Compostela, 1990.

9. S. Caenepeel and F. Van Oystaeyen, Brauer groups and the cohomology of graded rings, Dekker, New York, 1988.

10. S. Chase and A. Rosenberg, Amitsur cohomology and the Brauer group, Mem. Amer. Math. Soc. no. 52 (1965), 20-45.

11. S. U. Chase and M. E. Sweedler, Hopf algebras and Galois theory, Lecture Notes in Math., vol. 97, Springer-Verlag, Berlin, 1969.

12. L. N. Childs, The Brauer group of graded Azumaya algebras II: graded Galois extensions, Trans. Amer. Math. Soc. 204 (1975), 137-160. 
13. L. N. Childs, G. Garfinkel, and M. Orzech, The Brauer group of graded Azumaya algebras, Trans. Amer. Math. Soc. 175 (1973), 299-326.

14. A. P. Deegan, $A$ subgroup of the generalized Brauer group of $\Gamma$-Azumaya algebras, J. London Math. Soc. 23 (1981), 223-240.

15. F. R. DeMeyer, Galois theory in separable algebras over commutative rings, Illinois J. Math. 10 (1966), 287-295.

16. F. DeMeyer and T. Ford, Computing the Brauer-Long group of $\mathbf{Z} / 2$-dimodule algebras, $\mathrm{J}$. Pure Appl. Algebra 54 (1988), 197-208.

17. F. DeMeyer and E. Ingraham, Separable algebras over commutative rings, Lecture Notes in Math., vol. 181, Springer-Verlag, Berlin, 1971.

18. O. Gabber, Some theorems on Azumaya algebras, Groupes de Brauer, Lecture Notes in Math., vol. 844, Springer-Verlag, Berlin, 1981.

19. D. K. Harrison, Abelian extensions of commutative rings, Mem. Amer. Math. Soc. no. 52 (1965), 66-79.

20. M. A. Knus, Algebras graded by a group, Category Theory, Homology Theory, and Their Applications II, Lecture Notes in Math., vol. 92, Springer-Verlag, Berlin, 1969.

21. _ A Teichmüller cocycle for finite extensions (preprint).

22. M. A. Knus and M. Ojanguren, Théorie de la descente et algèbres d'Azumaya, Lecture Notes in Math., vol. 389, Springer-Verlag, Berlin, 1974.

23. F. Long, A generalization of the Brauer group of graded algebras, Proc. London Math. Soc. 29 (1974), 237-256.

24. __ The Brauer group of dimodule algebras, J. Algebra 30 (1974), 559-601.

25. __, Generalized Clifford algebras and dimodule algebras, J. London Math. Soc. (2) 13 (1976), 438-442.

26. J. S. Milne, Étale cohomology, Princeton Univ. Press, Princeton, N.J., 1980.

27. C. Nastasescu and F. Van Oystaeyen, Graded ring theory, Library of Math., vol. 28, NorthHolland, Amsterdam, 1982.

28. M. Orzech, On the Brauer group of algebras having a grading and an action, Canad. J. Math. 28 (1976), 533-552.

29. __ Correction to "On the Brauer group of algebras having a grading and an action," Canad. J. Math. 32 (1980), 1523-1524.

30. A. Rosenberg and D. Zelinsky, Automorphisms of separable algebras, Pacific J. Math. 11 (1961), 1109-1117.

31. C. Small, The Brauer-Wall group of a commutative ring, Trans. Amer. Math. Soc. 156 (1971), 455-491.

32. F. Tilborghs, The Brauer group of $R$-algebras which have compatible $G$-action and $\mathbf{Z} \times G$ grading, Comm. Algebra (to appear).

33. _ The Brauer-Long group of a field $R$ and the cyclic group $C_{p}$ (preprint).

34. __ Brauer groups of algebras with gradings and actions, Thesis, Free University of Brussels, 1989.

35. F. Tilborghs and F. Van Oystaeyen, Brauer-Wall algebras graded by $\mathbf{Z}_{2} \times \mathbf{Z}$, Comm. Algebra 16 (1988), 1457-1478.

36. O. E. Villamayor and D. Zelinsky, Brauer groups and Amitsur cohomology for general commutative ring extensions, J. Pure Appl. Algebra 10 (1977), 19-55.

37. C. T. C. Wall, Graded Brauer groups, J. Reine Angew. Math. 213 (1964), 187-199. GIUM

Faculty of Applied Sciences, Free University of Brussels, VUB B-1050 Brussels, Bel-

Department of Mathematics, Mount Allison University, Sackville, New Brunswick, CANADA E0A 3C0 